\title{
Assigning vibrational polyads using relative equilibria: application to ozone
}

\author{
I.N. Kozin ${ }^{\mathrm{a}, \mathrm{b}, 1}$, D.A. Sadovskiî́, ${ }^{*}$, B.I. Zhilinskií ${ }^{\mathrm{c}}$ \\ ${ }^{a}$ Mathematics Institute, University of Warwick, Coventry CV4 7AL, UK \\ ${ }^{\mathrm{b}}$ Department of Chemistry, University of Aberdeen, Meston Walk, Aberdeen AB24 3UE, UK \\ ${ }^{\mathrm{c}}$ Université du Littoral, UMR 8101 du CNRS, 59140 Dunkerque, France
}

Received 6 July 2004; received in revised form 30 September 2004; accepted 28 October 2004

\begin{abstract}
We demonstrate how relative equilibria of a vibrating molecule, which are families of principal periodic orbits otherwise known as nonlinear normal modes, can be used to describe the global polyad structure of vibrational energy levels. The classical action integral $n(E)$ computed along these orbits at different energies $E$ corresponds to the polyad quantum number $n$ so that the energy $E(n)$ of different relative equilibria describes the splitting of $n$-polyads. Further information on the internal polyad structure can be driven from the stability analysis of relative equilibria. We use the ozone molecule as a concrete example where $n$-polyads or "hyperpolyads" should be distinguished from the wellknown polyads of the 1:1 stretching mode resonance; the stretching polyads are structural elements of hyperpolyads. We give dynamical interpretation of the relation between relative equilibria and $n$-polyads based on the normal form reduction in the limit of small vibrations near the equilibrium.
\end{abstract}

(C) 2004 Elsevier B.V. All rights reserved.

PACS: 33.15.Mt; 33.20.Vq

Keywords: Relative equilibrium; Normal form; Periodic orbit; Vibrational polyad; Dynamical symmetry; Local mode

\section{Introduction}

In this article we intend to describe the global polyad structure of the vibrational levels of ozone. We consider the classical mechanical analogue of the vibrational molecular system and use the results of the qualitative analysis of this classical analogue in order to characterize the polyad structure of the original quantum system. After specifying the dynamical or polyad symmetry of our system, we find its relative equilibria (RE), which provide the framework of the qualitative study.

Definition of relative equilibrium. Consider a Hamiltonian dynamical system with phase space $P$ and Lie symme-

\footnotetext{
* Corresponding author. Tel.: +33 328658263; fax: +33328658263 .

E-mail addresses: i.kozin@dl.ac.uk (I.N. Kozin); sadovski@univ-littoral.fr (D.A. Sadovskii); zhilin@univ-littoral.fr (B.I. Zhilinskii).

${ }^{1}$ Present address: Department of Computational Science and Engineering, CCLRC Daresbury Laboratory, Keckwick Lane, Warrington WA4 4AD, UK.
}

try group $G$. Let $g_{t} \subseteq G$ (with parameter $t \in \mathbb{R}$ ) be a oneparameter continuous subgroup of $G$. A phase curve of our system which coincides with the group orbit of the action of $g_{t}$ on $P$ is called relative equilibrium (RE), see Appendix 5C of [1a] and Chapter 3.3 of [1b]. Reduction of the symmetry group $g_{t}$ maps such phase curves to equilibria of the reduced system.

We describe the RE of ozone first as "short" periodic orbits and use their action-energy diagram to describe the polyad structure. Subsequently, we normalize the classical system and uncover the explicit relation of RE to the polyad integral of the normal form. Finally, we quantize the normal form and compare the results to the ab initio energy levels.

\subsection{Rotational relative equilibria}

The most obvious molecular example of RE comes up in the study of free molecule rotation. In this case, since the 
system is invariant with regard to rotations of the laboratory fixed frame, $G=\mathrm{SO}(3), g_{t}=\mathrm{SO}(2)$, and RE correspond to rotation about stationary axes [1a,2]. Due to additional finite symmetries, the molecule has usually several equivalent stationary axes. Molecular spectroscopists have recognized long ago that such axes manifest themselves in the quantum spectrum as level clusters [3]. Applications [4,5] normally study the reduced system, for which stationary axes of rotation become equilibria. For each non-zero value of the length $j$ of the total angular momentum, the reduced phase space is a 2-sphere $\mathbb{S}_{j}^{2}$. The reduced Hamiltonian is a function on this space. It is often called rotational energy surface [6] and is depicted as a deformed sphere whose maxima, minima, or saddle points represent RE.

\subsection{Vibrational relative equilibria}

We can extend our definition of RE by allowing that the symmetry $G$ is not necessarily strict, but is an approximate dynamical symmetry. This extension is particularly useful in the study of molecular vibrations.

\subsubsection{Resonances}

The molecular vibrational Hamiltonian in the case of small vibrations about a well-defined molecular equilibrium (the case of "rigid" molecules) is a power series

$H=H^{(0)}+\epsilon H^{(1)}+\epsilon^{2} H^{(2)}+\cdots$

in displacements $q_{k}$ and conjugate momenta $p_{k}$, whose terms can be distinguished by the uniform smallness parameter $\epsilon$. The quadratic part of $H$ is

$H^{(0)}=\frac{1}{2} \sum_{k=1}^{K} \omega_{k}\left(q_{k}^{2}+p_{k}^{2}\right)=\sum_{k=1}^{K} \omega_{k} n_{k}$,

where $K$ is the number of vibrational degrees of freedom and frequencies $\omega_{1}: \omega_{2}: \cdots: \omega_{K}$ obey, approximately or exactly, the resonance condition $m_{1}: m_{2}: \cdots: m_{K}$, where $m_{k}$ are positive integers. (Note that here $n_{k}$ are classical actions which quantize as $n_{k} \rightarrow N_{k}+\frac{1}{2}$, where $N_{k}=0,1,2$, etc.) The resonance condition defines $G$. For example, condition $1: 1: \cdots: 1$ results in $G=\operatorname{SU}(K)$. The subgroup $g_{t}$ is given by the flow $\varphi_{t}$ of the vector field $X_{n}$ of the Hamiltonian

$n=\frac{1}{2} \sum_{k=1}^{K} m_{k}\left(q_{k}^{2}+p_{k}^{2}\right)$.

Comparing $H^{(0)}$ and $n$, we note that frequencies $\omega_{k}$ in the linearized Hamiltonian $H^{(0)}$ are approximated in $n$ by integers $m_{k}$. Furthermore, it is often convenient to rescale energy so, that $H^{(0)} \omega^{-1} \approx n$, where $\omega$ is the mean characteristic vibrational frequency of the molecule. We also find from the equations of motion for the system with Hamiltonian $n$ that $\varphi_{t}$ acts as a rotation on the phase space $\mathbb{R}_{q, p}^{2 K}$.

\subsubsection{Reduction of dynamical symmetry}

In general, the Poisson bracket $\left\{H^{(s)}, n\right\}$ does not vanish unless $s=0$. We assume, however, that the higher orders $H^{(s)}$ are approximately $G$-invariant. More precisely, assuming that $\left\{H^{(s)}, n\right\}$ for $s>0$ are small, we can normalize $H$ so that all terms $\mathcal{H}^{(s)}$ in the transformed Hamiltonian $\mathcal{H}$ Poisson commute with $n$. The normal form $\mathcal{H}$ is, therefore, strictly $G$-invariant. The canonical transformation $\mathcal{L}_{\epsilon}: H \rightarrow \mathcal{H}$ is a near unity transformation which becomes identity when $\epsilon \rightarrow 0$ [7]; in particular, $\mathcal{H}^{(0)}=H^{(0)}$. Like the original Hamiltonian $H$, the normal form $\mathcal{H}$ is an $\epsilon$-series. The most practical and direct method of normalizing such series is the Lie series method [8a-d]. In most cases with $K>1$, the series $\mathcal{H}$ diverges when taken to unreasonably high orders (see, for example, Appendix 7 of [1a]). Consequently, we have to truncate $\mathcal{H}$ and specify conditions at which such truncated normal form is useful. Normally we restrict the energy $H$, the value of $n$, and/or the perturbation scale $\epsilon$.

By construction, the Hamiltonian function $n$ is an integral of motion of the normalized system. We can, therefore, reduce this system at each given value of $n>0$, so that the value of $n$ becomes a parameter. The reduced Hamiltonian $\mathcal{H}_{n}$ is the normal form $\mathcal{H}$ expressed as a function on the reduced phase space $P_{n}$.

In the case of the resonance condition $1: 1: \cdots: 1$, the reduced phase space is the complex projective space $\mathbb{C} P^{K-1}$. Dynamical variables of the reduced system are quadratic polynomials in $(q, p)$ which Poisson commute with $n$ and generate a Poisson algebra $\operatorname{su}(K)$. In this paper we will encounter spaces $\mathbb{C} P^{2}$ and $\mathbb{C} P^{1}$.

The reduced phase space of the two-mode system $(K=2)$ with resonance $1: 1$ is a 2 -sphere $\mathbb{S}^{2}$ which is isomorphic to $\mathbb{C} P^{1}$ [9]. This basic case has, of course, been studied in great detail, notably in application to the Hénon-Heiles system [10] and its molecular analogues [11], and 1:1 resonant vibrational subsystems of polyatomic molecules [12]. Since the reduced system is equivalent to the reduced rotational system (see Appendix A.4), we use the analysis described briefly in Section 1.1.

\subsubsection{Polyads and polyad Hamiltonians}

The term polyad quantum number is now widely used in molecular spectroscopy to label a relatively isolated aggregation of vibrational states [13]. For example, two stretching modes of an $\mathrm{AB}_{2}$ molecule often have nearly the same frequencies $\omega_{1}$ and $\omega_{3}$ and can, therefore, be considered as a 1:1 system mentioned above. A stretching polyad of such molecule is labeled by $n_{s}=n_{1}+n_{3}$, where $n_{1}$ and $n_{3}$ are numbers of quanta in each of the stretching modes. The polyad number can be extended to include the bending vibration as well. Frequently, there is a near 1:2 resonance between stretching and bending modes. Then the polyad number can 
be defined as

$N_{1: \frac{1}{2}: 1}=n_{1}+\frac{1}{2} n_{2}+n_{3}$

Such number is often used for triatomic molecules [14]. If the normal mode frequencies of $\mathrm{AB}_{2}$ can be approximated by integers $k_{1}: k_{2}: k_{3}$, we can use the polyad number

$N_{k_{1}: k_{2}: k_{3}}=k_{1} n_{1}+k_{2} n_{2}+k_{3} n_{3}$.

According to this general definition, the number $N_{2: 1: 2}$ should be used instead of the above $N_{1: \frac{1}{2}: 1}$.

In this work, we will use the polyad number (cf. Appendix A.1)

$n=N_{1: 1: 1}=n_{1}+n_{2}+n_{3}$,

which we call the hyperpolyad number. We propose to classify quantum states first using $n$ and then, if possible, other quantum numbers. This principle works very well in the ozone molecule where all known assigned vibrational levels $[15 \mathrm{a}, \mathrm{b}]^{2}$ can be easily and unambiguously grouped into $n$-polyads, even though the resonance condition for ozone is much closer to $5: 3: 5$ or $2: 1: 2$ than to plain $1: 1: 1$.

Polyad Hamiltonians are used in spectroscopy to describe internal structure of polyads. These spectroscopic Hamiltonians are called effective and model to emphasize the absence of explicit inter-polyad interaction terms and the liberty in the choice of resonance condition, respectively. Parameters of the polyad Hamiltonian are often treated as phenomenological spectroscopic constants, whose values are obtained by fitting experimental data. To further confuse the uninitiated, typical resonance conditions (=models) and related polyad Hamiltonians are traditionally named after Fermi, DarlingDennyson, and others [16]. The simplest example is again the 1:1 Hamiltonian, which is analogous to an effective rotational Hamiltonian. (Recall that the "rotational polyad" is a multiplet of levels with the same angular momentum quantum number $J$, see Appendix A.4 and [17].)

Going back to Section 1.2.2 we can see that (i) the polyad approximation amounts to the dynamical symmetry assumption given by the resonance condition and followed by reduction, (ii) polyad Hamiltonians are nothing but reduced Hamiltonians $\mathcal{H}_{n}$, (iii) vibrational dynamics of the reduced system defines internal polyad structure. Spectroscopists construct quantum polyad Hamiltonians $\hat{H}_{n}$ using terms which preserve the polyad number. In order to commute with quantum operator $\hat{n}$, terms in $\hat{H}_{n}$ are restricted to have creationannihilation operators of certain degree and type. This is analogous to the classical construction of the ring of the dynamical invariants (see Appendix A.2).

\footnotetext{
${ }^{2}$ The work [15b] gives essentially almost the same potential as in [15a]. The latter was obtained using MORBID, which made a few approximations in the kinetic energy operator. These were "absorbed" in the potential. The new paper removed this deficiency using the "Exact Kinetic Energy" operator.
}

\subsubsection{Modes and periodic orbits}

The spectroscopic concept of "mode" is often confusingly vague. The common practice of opposing "local" and "normal" modes can be a good example. The concept of RE helps to bring the situation in better order.

Consider the standard bound molecular system of small vibrations described in terms of small displacements $q$ and corresponding conjugate momenta $p$. The zero-order vibrational Hamiltonian is a sum of two positively definite quadratic forms, the kinetic energy $T$ and potential $V$. The phase space variables $(q, p)$ can be chosen so that both $T(p)$ and $V(q)$ are diagonal. Such variables correspond to normal modes [18]. The presence of symmetry often simplifies the task of diagonalization. Thus ozone and molecules $\mathrm{AB}_{2}$ have only one asymmetric displacement $q_{3}$ which defines the normal mode $v_{3}$ unambiguously. The form of the two symmetric modes $v_{1}$ and $v_{2}$ depends on the particular $T(p)$ and $V(q)$.

The dynamical concept of mode begins with the theorem of Weinstein [19]. Consider a stable equilibrium of a Hamiltonian system with $K$ degrees of freedom, and suppose that harmonic frequencies are incommensurate, i.e., there are no resonances. Then near the limit of linearization, i.e., at energies $h$ close to the equilibrium energy $h_{0}$, the system has a set of $K$ energy-dependent families of periodic trajectories which are defined by the nonlinear terms of the local Hamiltonian. These families are basic vibrational modes of the system near the given equilibrium. To distinguish them from the $K$ normal modes defined above, it was suggested to use the term nonlinear normal modes [20a-c]. We realize immediately that, in fact, these modes are nothing but vibrational RE.

In a resonant system, the number of nonlinear normal modes $(=\mathrm{RE})$ can be a priori greater than the number of normal modes (coordinates) [20a-c]. Again, the presence of symmetry can greatly simplify the task of characterizing these RE. Thus recall the textbook example of the eight RE of the two-dimensional Hénon-Heiles system [10,20a-c], a nonlinear 1:1 resonant oscillator with symmetry $D_{3}$, and its molecular analogue-the $\mathrm{H}_{3}^{+}$molecular ion [11].

As the energy $h$ gets further from $h_{0}$, nonlinear normal modes $(=\mathrm{RE})$ can bifurcate, and in particular, their number can increase. These bifurcations correspond to bifurcations of the equilibria of the reduced system. The new (families of) periodic trajectories are, therefore, also RE. Different sets of RE correspond to qualitatively different internal polyad structures. The classic example of an RE-bifurcation is the so-called normal-to-local mode transition, which happens in ozone when $h$ is very close to $h_{0}$. We will discuss this bifurcation in detail.

At very low energies, the ozone molecule has three nonlinear normal modes which correlate with the three normal modes, symmetric and antisymmetric stretching $v_{1}$ and $v_{3}$, and bending $\nu_{2}$. At slightly higher energies, it also has two extra equivalent RE, which spectroscopists call "bond length" modes or local modes [21]. These modes are close to the vibration of the individual $\mathrm{O}-\mathrm{O}$ bonds. 
Like any stable periodic trajectories, stable RE of period $T_{\mathrm{RE}}(n)$ can undergo period- $s$ bifurcations which involve trajectories of period $s T_{\mathrm{RE}}(n)$. Within the framework of the polyad approximation, we can only describe period-1 bifurcations. Such bifurcations affect the internal structure of the hyperpolyad but not its validity. Cascading higher-period bifurcations signal the destruction of hyperpolyads and the onset of chaos. However, when suggesting the limits of the hyperpolyad approximation for the classical system, we should also consider that the quantum analogue system is, generally, more robust to chaos, and our approximation has a good chance to stretch further than we would expect classically.

\subsubsection{Assignment of quantum states}

Classical textbooks on molecular vibrations and spectroscopy $[16,18]$ usually suggest normal modes for vibrational energy level assignment. More recently, the local modes of a number of hydrogen-bonded molecules and ozone were suggested as more "physical" [21]. On the other hand, both nomenclatures can be considered formally equivalent in the limit of small distortions where local and normal modes are linear combinations of each other and there is linear relationship between the corresponding quantum numbers. The current spectroscopic practice is to present both local and normal mode quantum numbers and specify their relationship.

Dynamical approach to assigning quantum states is based on localization near RE or, in general, other dynamically invariant subspaces. Consider a sufficiently stable relative equilibrium $\Pi$, such as the local mode RE of ozone. Take a projection $\Pi_{q}$ of the periodic orbit $\Pi$ in the original phase space $\mathbb{R}_{(q, p)}^{2 K}$ on the configuration space $\mathbb{R}_{q}^{K}$. When a quantum wavefunction is localized near $\Pi_{q}$, its nodes follow $\Pi_{q}$. In the limiting case the number of such nodes $N_{\Pi}$ equals the polyad quantum number $N$. When $N_{\Pi}$ is less than $N$, other degrees of freedom are involved in the direction transversal to $\Pi$. Yet, if $N_{\Pi}$ is sufficiently close to $N$, the node pattern can still remain a regular lattice which follows $\Pi$. For such states $N_{\Pi}$ is clearly a good quantum number. Of course, not all states fall into such category. Other states can, possibly, be assigned in terms of other stable RE, and some strongly delocalized states would remain without any meaningful dynamical assignment except for the hyperpolyad number $N$. Thus, our main proposition is to begin with the hyperpolyad assignment of all states, and then classify them further where it is possible.

\subsection{Outline}

The paper has three main directions. In order to uncover the existence of hyperpolyads, we first study numerically (see Section 3) the main periodic orbits of the vibrational system of ozone with Hamiltonian in [15a,b]. We then show the relation of these orbits to relative equilibria. We normalize the initial three-mode Hamiltonian of [15a] and analyze RE as equilibria of the reduced system (Section 4). Finally we compute quantum levels in two ways, using local linearization near stable periodic orbits (Section 3.3) and by quantization of the global normal form (Section 4.5). We reproduce adequately all quantum levels assigned in $[15 \mathrm{a}-\mathrm{c}]$ and thus demonstrate the validity of the polyad approximation. Subsequently, we address the main problem faced in [15a,b,22], namely the assignment of quantum levels computed numerically. We suggest a direct method of computing the hyperpolyad number for a given wavefunction (Section 5).

This work should, of course, be regarded in the context of numerous publications on the vibrational levels and dynamics of triatomic and polyatomic molecules [23,24a-f], including more recent work on ozone [15a-c,25,26]. In particular, we like to mention the work by Lu and Kellman [14], who study ozone on the basis of a 2:1:2 model polyad Hamiltonian. They focus mainly on stretching polyads (cf. Section 4.3) and apply the standard analysis based on the angular momentum analogy $[6,12,5]$.

Contrary to [14], we derive our polyad approximation from the full vibrational Hamiltonian, which describes all experimentally known states of ozone. The same computation was independently attempted by Joyeux [27], who obtained even better reproduction of the numerical quantum energies of [15a,b]. However, far from trying to compete with [15ac] in accuracy of reproduced quantum energies, we consider our classical study and the subsequent quantization as a basic qualitative tool of dynamical characterization of computed states. We like to focus mostly on the global hyperpolyad structure and on the problem of level assignment.

\section{Ozone molecule}

The most abundant isotopomer of ozone molecule has three identical atoms and isosceles equilibrium configuration with two equal bond lengths $r_{12}=r_{23}=r_{e}$ and the bond angle $\alpha_{e}$. Vibrations of this molecule are described most naturally in terms of two dimensionless bond length displacements $\xi_{1}$ and $\xi_{2}$, such that

$r_{12}=r_{e}\left(1+\xi_{1}\right), \quad r_{23}=r_{e}\left(1+\xi_{2}\right)$,

and the bond angle displacement $\alpha$. In these coordinates, the kinetic energy term $T$ in the Hamiltonian

$$
H=\frac{1}{m r_{e}^{2}} T\left(\xi, \eta, \alpha, p_{\alpha}\right)+V\left(\xi_{1}, \alpha, \xi_{2}\right)
$$

has the form

$$
\begin{aligned}
T= & \eta_{1}^{2}+\eta_{2}^{2}+\frac{\left(1+\xi_{1}\right)^{2}+\left(1+\xi_{2}\right)^{2}-\left(1+\xi_{1}\right)\left(1+\xi_{2}\right) \cos \left(\alpha_{e}+\alpha\right)}{\left(1+\xi_{1}\right)^{2}\left(1+\xi_{2}\right)^{2}} p_{\alpha}^{2}+\cos \left(\alpha_{e}+\alpha\right) \eta_{1} \eta_{2} \\
& -\frac{\sin \left(\alpha_{e}+\alpha\right)}{\left(1+\xi_{1}\right)\left(1+\xi_{2}\right)} p_{\alpha}\left\{\left(1+\xi_{1}\right) \eta_{1}+\left(1+\xi_{2}\right) \eta_{2}\right\}
\end{aligned}
$$


where $\eta_{1}, \eta_{2}$, and $p_{\alpha}$ are momenta conjugate to $\xi_{1}, \xi_{2}$, and $\alpha$, respectively. The potential function $V\left(\xi_{1}, \alpha, \xi_{2}\right)$ was determined very accurately in the recent work by Tyuterev et al. [15a] from fitting all known experimental data. This potential has good global properties and the dissociation energy of $9450 \mathrm{~cm}^{-1}$ which is in good agreement with experiment. An improvement of this potential was reported later in [15b].

The Hamiltonian (2) is invariant with respect to bond permutation $C_{2}$ and time reversal $\mathcal{T}$. These two finite symmetry operations generate a group of order four with structure $Z_{2} \times Z_{2}$. Appendix $\mathrm{B}$ gives a detailed description of this group and of its implications.

For the normalization purposes we represent the Hamiltonian (2) as a power series $H(q, p)$ in the near equilibrium normal mode displacements $q=\left(q_{1}, q_{2}, q_{3}\right)$ and conjugate momenta $p=\left(p_{1}, p_{2}, p_{3}\right)$

$$
\begin{aligned}
H= & \frac{\omega_{1}}{2}\left(p_{1}^{2}+q_{1}^{2}\right)+\frac{\omega_{2}}{2}\left(p_{2}^{2}+q_{2}^{2}\right)+\frac{\omega_{3}}{2}\left(p_{3}^{2}+q_{3}^{2}\right) \\
& +\epsilon H_{1}+\epsilon^{2} H_{2}+\epsilon^{3} H_{3}+\cdots .
\end{aligned}
$$

The totally symmetric coordinates $q_{1}$ and $q_{2}$ depend on $\xi_{1}+\xi_{2}$ and $\alpha$; the symmetric stretch $q_{1}$ has a predominant contribution due to $\xi_{1}+\xi_{2}$ while the bending coordinate $q_{2}$ depends mostly on $\alpha$. The anti-symmetric stretch coordinate $q_{3}$ is proportional to $\left(\xi_{1}-\xi_{2}\right)$. The zero order term in the series $H(q, p)$ is in the standard diagonal quadratic form. Note, that unlike in some other $\mathrm{AB}_{2}$ molecules, such as water, the symmetric stretch frequency $\omega_{1}$ is larger than that of the asymmetric stretch. In this study we expanded $H(q, p)$ to degree 6 (order $\epsilon^{4}$ ); cubic and quartic terms in this series are listed in Table 1.

\section{Relative equilibria as principal periodic orbits}

We begin with direct numerical study of periodic orbits which correspond to relative equilibria (RE). As follows from Section 1.2, we should be interested primarily in the energy-action characteristics of these orbits. Indeed, action corresponds to classical polyad number $n$ and the polyad structure is given by the RE energies at fixed $n$. We argue that our periodic orbit analysis justifies the basis for using the 1:1:1 resonance model. This agrees with Lu and Kellman [14], who did not find low energy bifurcations in their 2:1:2 model except for the local mode bifurcation, which can be equally well described by the $1: 1: 1$ model.

\subsection{Energy-action characteristics}

Continuation of periodic orbits of molecular systems has been done by a number of authors, notably Prosmiti and Farantos [28]. We do it in a somewhat different context [29]. We opted for the very well-developed continuation package
Table 1

Hamiltonian of ozone with vibrational potential of Ref. [15a] expressed

\begin{tabular}{|c|c|c|c|c|}
\hline Order & Coefficient & Term & Coefficient & Term \\
\hline \multirow[t]{3}{*}{$\overline{\epsilon^{0}}$} & 1132.3600 & $\frac{1}{2}\left(q_{1}^{2}+p_{1}^{2}\right)$ & \multicolumn{2}{|c|}{ Symmetric stretch } \\
\hline & 714.6150 & $\frac{1}{2}\left(q_{2}^{2}+p_{2}^{2}\right)$ & \multicolumn{2}{|c|}{ Bending } \\
\hline & 1086.9425 & $\frac{1}{2}\left(q_{3}^{2}+p_{3}^{2}\right)$ & \multicolumn{2}{|c|}{ Asymmetric stretch } \\
\hline \multirow[t]{8}{*}{$\epsilon^{1}$} & -40.26499 & $q_{1}^{3}$ & -10.30595 & $q_{1} p_{3}^{2}$ \\
\hline & 29.41346 & $q_{2}^{3}$ & 20.49931 & $q_{2} p_{1}^{2}$ \\
\hline & -8.49418 & $q_{1} q_{2}^{2}$ & -5.25573 & $q_{2} p_{2}^{2}$ \\
\hline & -195.22643 & $q_{1} q_{3}^{2}$ & -13.48096 & $q_{2} p_{3}^{2}$ \\
\hline & 8.92844 & $q_{1}^{2} q_{2}$ & -41.43463 & $q_{1} p_{1} p_{2}$ \\
\hline & 65.82223 & $q_{2} q_{3}^{2}$ & -11.77458 & $q_{2} p_{1} p_{2}$ \\
\hline & 6.36668 & $q_{1} p_{1}^{2}$ & 13.26030 & $q_{3} p_{2} p_{3}$ \\
\hline & -24.45093 & $q_{1} p_{2}^{2}$ & 4.98473 & $q_{3} p_{1} p_{3}$ \\
\hline \multirow[t]{14}{*}{$\epsilon^{2}$} & 0.23935 & $q_{1}^{4}$ & 0.16508 & $q_{1}^{2} p_{1}^{2}$ \\
\hline & 2.21248 & $q_{2}^{4}$ & -0.03366 & $q_{2}^{2} p_{1}^{2}$ \\
\hline & 4.36140 & $q_{3}^{4}$ & 0.39659 & $q_{2}^{2} p_{2}^{2}$ \\
\hline & -4.01685 & $q_{1}^{3} q_{2}$ & -0.35362 & $q_{1} q_{2} p_{3}^{2}$ \\
\hline & -1.56543 & $q_{1} q_{2}^{3}$ & -2.29488 & $q_{1} q_{2} p_{1} p_{2}$ \\
\hline & -0.77267 & $q_{1}^{2} q_{2}^{2}$ & -0.15429 & $q_{1} q_{3} p_{1} p_{3}$ \\
\hline & 12.92855 & $q_{1}^{2} q_{3}^{2}$ & -1.04417 & $q_{2}^{2} p_{1} p_{2}$ \\
\hline & -13.31552 & $q_{1} q_{2} q_{3}^{2}$ & 1.58181 & $q_{3}^{2} p_{1} p_{2}$ \\
\hline & -0.26197 & $q_{2}^{2} q_{3}^{2}$ & 0.94469 & $q_{1} q_{2} p_{2}^{2}$ \\
\hline & 1.19930 & $q_{3}^{2} p_{2}^{2}$ & -0.36182 & $q_{1} q_{2} p_{1}^{2}$ \\
\hline & 1.80520 & $q_{1}^{2} p_{2}^{2}$ & 0.47273 & $q_{1}^{2} p_{1} p_{2}$ \\
\hline & -0.13517 & $q_{1}^{2} p_{3}^{2}$ & 0.27819 & $q_{2} q_{3} p_{1} p_{3}$ \\
\hline & -0.23128 & $q_{2}^{2} p_{3}^{2}$ & 0.74005 & $q_{2} q_{3} p_{2} p_{3}$ \\
\hline & 0.42515 & $q_{3}^{2} p_{1}^{2}$ & -0.41046 & $q_{1} q_{3} p_{2} p_{3}$ \\
\hline
\end{tabular}
using normal mode coordinates

CONTENT [30], ${ }^{3}$ which can be easily adapted for our purposes by introducing a free dummy parameter $\lambda$ in the equations of motion

$\dot{q}_{i}=\frac{\partial H}{\partial p_{i}}, \quad \dot{p}_{i}=-\frac{\partial H}{\partial q_{i}}+\lambda p_{i}, \quad i=1,2,3$.

We normally start from a normal mode and continue the periodic orbit by allowing the period to change and calculating the energy and action. The parameter $\lambda$ is also varied but maintained around zero. In this way one is able to produce a graph of the energy against the action for every periodic orbit, see Fig. 1.

\subsection{Bifurcation of periodic orbits}

At very low energies, the three periodic orbits (POs) correlate with the three normal modes, see Fig. 1; the bending PO has the minimum energy at given fixed action $n$, the symmetric stretch PO stays on top, and the asymmetric stretch PO

\footnotetext{
3 This package is based on the other versatile continuation package AUTO and provides extremely flexible and powerful graphic interface.
} 


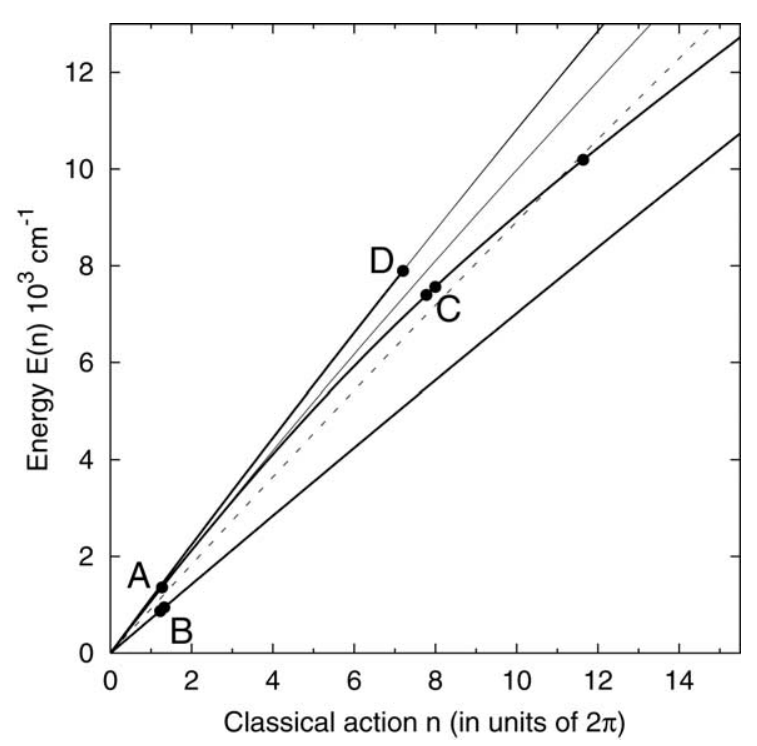

Fig. 1. Action-energy diagram for principal periodic orbits (PO) of ozone. Bold and fine lines indicate stable and unstable PO, respectively; circles denote bifurcations; dotted line shows the average polyad energy $E_{0}(n)$, which is subtracted in subsequent figures.

lies slightly below it. The first bifurcation, which happens in ozone, is the well-known bifurcation of the asymmetric stretch PO resulting in normal-to-local mode transition [14]. This bifurcation is indicated by point A in Fig. 1 with energy $E=1336 \mathrm{~cm}^{-1}$ and action $n=1.24$, which is even below the ground state energy of $1451 \mathrm{~cm}^{-1}$. The asymmetric stretch PO loses stability and the two stable equivalent local-mode PO branch out. The bifurcation breaks the bond permutation symmetry $C_{2}$. The two local-mode PO remain symmetric with regard to the time reversal symmetry $\mathcal{T}$ but are mapped into each other by $C_{2}$. Continuation of the unstable PO and the two new POs is shown in Fig. 1 by a fine line and a single bold line, respectively. As the action $n$ increases further, the energy separation between the unstable asymmetric stretch PO and the local-mode POs increases, stability of the two local-mode POs grows and quantum localization near them becomes possible. Since these POs are equivalent due to the $C_{2}$ symmetry of the system, the localized quantum states form doublets which are readily observed experimentally.

Next, there are two pairs of period doubling bifurcations. Point $\mathrm{B}$ corresponds to the sequence of two bifurcations of the bending $\mathrm{PO}$, while point $\mathrm{C}$ marks the two bifurcations of the local-mode PO. In these bifurcations, each PO becomes briefly unstable thus bringing in some irregularity in the dynamics. It is hard to judge from the limited analysis given here about the full impact of these bifurcations. It seems that they are unimportant to the global polyad structure. This will be further justified by the comparison to quantum levels.

Analyzing the energy-action diagram in Fig. 1 further, we observe a bifurcation of the symmetric stretch $\mathrm{PO}$ (point D) at $E=10857 \mathrm{~cm}^{-1}$ and $n=10.04$. This is a pitchfork bifurcation which produces two new equivalent POs while the parent PO loses stability. However, unlike in the case of the local modes, the child branches stay so close to the parent that they can be hardly distinguished in Fig. 1. Another difference is that this bifurcation breaks the $\mathcal{T}$ symmetry and not $C_{2}$. The two new PO remain symmetric with regard to $C_{2}$ but are mapped into each other by $\mathcal{T}$.

Comparing these results to a computation with a more recent potential [15b], shows that the system of POs remains essentially unchanged (see below, energy $E$ in $\mathrm{cm}^{-1}$ and action $n$ ).

\begin{tabular}{lcrcc}
\hline Point & Ref. [15a] & \multicolumn{3}{c}{ Ref. [15b] } \\
\hline A & 1336 & 1.24 & 1361 & 1.27 \\
B & 898 & 1.26 & 872 & 1.22 \\
& 973 & 1.36 & 944 & 1.32 \\
C & 7454 & 7.83 & 7396 & 7.78 \\
& 7774 & 8.23 & 7564 & 7.99 \\
D & 10957 & 10.04 & 7893 & 7.20 \\
\hline
\end{tabular}

Nevertheless, we can see that the energy of point $\mathrm{D}$ varies largely even for a slightly different potential [15b]. Such sensitivity may point to compromised global high-energy properties of potentials [15a,b], and makes continuation into higher energy-action values questionable.

To summarize, we detected a number of bifurcations of "basic" periodic orbits of ozone, which can potentially damage the polyad approximation. However, most of them are of very local character (B and C) and are unlikely to be pronounced in the quantum spectrum, or happen very high in energy. The general conclusion is that the onset of chaos and the breakdown of our global 1:1:1 hyperpolyad approximation should happen for $n>10$; classical dynamics is sufficiently regular (most of the tori are still present) at lower $n$ and corresponding energies.

\subsection{Local quantization near stable RE}

Quantum states localized near a stable periodic orbit (PO) can be characterized using a local approximation of our Hamiltonian near the orbit. We can do this even when normalization is impossible, i.e., when the polyad approximation is no longer valid. In the simplest case, we consider the ground state of the harmonic approximation, i.e., the most localized state described in Section 1.2.5.

Computing harmonic frequencies $\left(\omega^{\prime}, \omega^{\prime \prime}\right)$ of oscillations in other degrees of freedom about the "central" PO comes at no additional cost because continuation programs, such as CONTENT, return the eigenvalues of the monodromy matrix and the stability indexes $\left(s^{\prime}, s^{\prime \prime}\right)$ of the PO. We can, therefore, find the estimates of harmonic frequencies in the directions transversal to the PO. Note that $\left(\omega^{\prime}, \omega^{\prime \prime}\right)$ for a stable PO are real numbers whose signs are given by the stability indexes +1 or -1 .

Quantization in this harmonic approximation is straightforward. The action integral along the PO equals $n_{0}+\mu$, 
Table 2

Energies $E_{\mathrm{RE}}$ in $\mathrm{cm}^{-1}$ of purely bending quantum states $\left(0, v_{2}, 0\right)$ of ozone computed using the harmonic approximation near the bending RE

\begin{tabular}{lccccc}
\hline$v_{2}$ & $E_{\mathrm{RE}}$ & $\Delta$ & $v_{2}$ & $E_{\mathrm{RE}}$ & $\Delta$ \\
\hline 1 & 701 & 0 & 6 & 4164 & \\
2 & 1399 & 0 & 7 & 4847 & \\
3 & 2095 & 0 & 8 & 5527 & 2 \\
4 & 2788 & 0 & 9 & 6202 & \\
5 & 3477 & 1 & 10 & 6874 & \\
\hline
\end{tabular}

$\Delta: E_{\mathrm{RE}}-E_{\mathrm{obs}}$ for the energies $E_{\mathrm{obs}}$ of the assigned quantum levels in [15a].

where the Maslov's correction $\mu$ for all RE is $\frac{1}{2}$ and does not change throughout the range of action-energies we consider. Actions for the two transversal oscillations are $n^{\prime}+\frac{1}{2}$ and $n^{\prime \prime}+\frac{1}{2}$. Here $n_{0}, n^{\prime}, n^{\prime \prime}$ are integers, and $n^{\prime}$ and $n^{\prime \prime}$ are small. This defines the EBK tori situated near the PO, and the energy of the corresponding quantum states

$E\left(n_{0}, n^{\prime}, n^{\prime \prime}\right)=E_{\mathrm{PO}}\left(n_{0}+\frac{1}{2}\right)+\omega^{\prime}\left(n^{\prime}+\frac{1}{2}\right)+\omega^{\prime \prime}\left(n^{\prime \prime}+\frac{1}{2}\right)$,

where $E_{\mathrm{PO}}$ is the energy-action characteristics of the PO in question.

Results of such quantization for the bending PO are compared to the assigned quantum levels from [15a] in Table 2. These levels lie just above the bending PO energy (lowest) in the energy-action diagram, see Figs. 1 and 2. Agreement for other POs is less satisfactory. Our simple approximation cannot account for tunneling in the case of the local mode PO, and we can only predict the average (unsplitted) energy. The symmetric stretch PO at high $n$ seems to be strongly affected by bifurcation $\mathrm{D}$ and the resulting presence of close satellite PO (see Fig. 1). This makes our harmonic approximation ineffective since at least one of the transversal motions is, obviously, very anharmonic. Nevertheless, Table 2 shows that it is possible to relate relative equilibria, periodic orbits, and quantum levels and then continue to higher energies.

\subsection{Hyperpolyad classification of quantum states}

In the previous section, we saw how certain localized states correlate with RE. Here we like to globalize the relation of the energy-action diagram for RE and the quantum energy spectrum of the system. This provides motivation for the rest of the paper and its appendices.

Conventionally, the normal mode quantum numbers are $\left(v_{1}, v_{2}, v_{3}\right)$, where subscripts correspond to our normal modes. We take all quantum states for which normal mode assignment $\left(v_{1}, v_{2}, v_{3}\right)$ was determined in [15a] and compute the hyperpolyad quantum number

$N=v_{1}+v_{2}+v_{3}$

as defined in (1). This number and the classical action integral $n_{\text {classical }}$ computed for each RE are related by the standard 3-

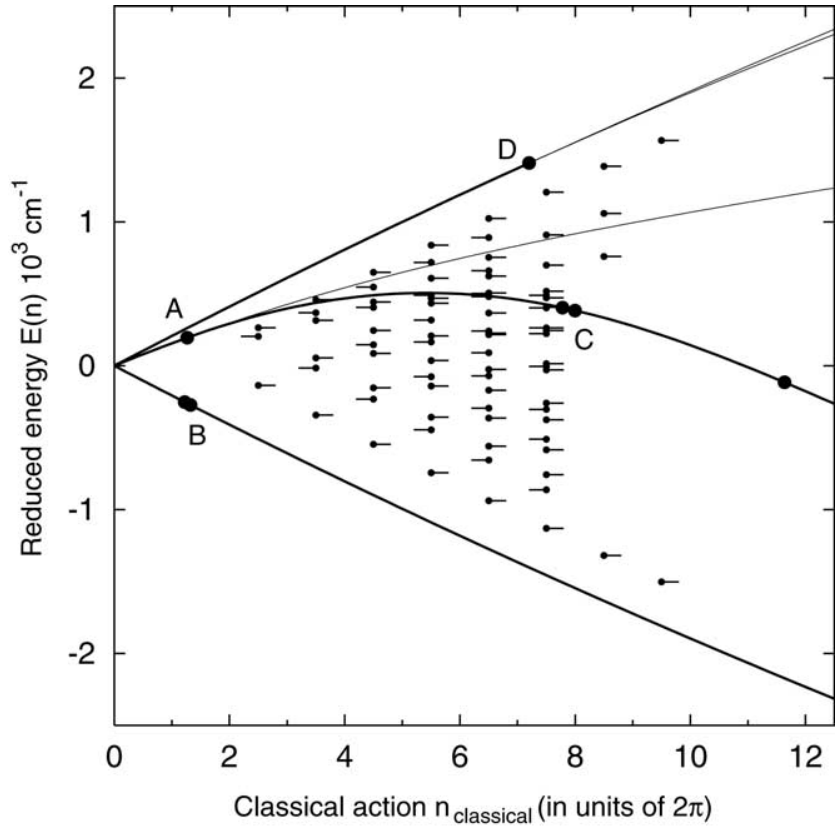

Fig. 2. Energies of periodic orbits (PO) and assigned quantum levels of ozone. Bold and fine solid lines indicate stable and unstable PO, respectively; circles mark bifurcations. Quantum levels are computed in [15a]; left- and rightward dashes correspond to symmetric and antisymmetric levels. Comparing to Fig. 1, note that the average polyad energy $E_{0}(n)$ is subtracted from $E(n)$.

oscillator quantization rule

$n_{\text {classical }}=\frac{1}{2 \pi} \oint p \mathrm{~d} q=v_{1}+v_{2}+v_{3}+\frac{3}{2}$.

Such polyad quantization is compared to the "local" nearRE scheme of the previous section in Appendix E. We can now superimpose quantum energy levels and the classical energy-action diagram of periodic orbits as shown in Fig. 2. We see immediately that the energy of RE's embraces the quantum spectrum correctly and that the latter exhibits a clear hyperpolyad structure.

We should also point out that assignment of the polyad number $n$ does not have to rely on the normal mode assignments $\left(v_{1}, v_{2}, v_{3}\right)$. In fact, the hyperpolyad assignment can remain physical even when the normal mode assignment is impossible. This is discussed in Section 5, where an alternative way of computing and assigning the value of the hyperpolyad quantum number $N$ is proposed.

\section{Relative equilibria as equilibria of reduced classical Hamiltonian}

We now follow the approach reviewed in Section 1.2 and uncover the relation of the periodic orbits studied in the previous section to the reduced system. Necessary details of reduction and normal form analysis are presented in Appendix A. 
Table 3

Terms in the first normalized Hamiltonian $\mathcal{H}_{n}$ describing hyperpolyads of the ozone molecule; notation is explained in Appendix A

\begin{tabular}{llllll}
\hline$\epsilon^{2}$ & & $\epsilon^{4}$ & & $\epsilon^{4}$ & \\
\hline-2.84040 & $n^{2}$ & -0.06479 & $x_{3}^{2} n$ & +0.63312 & $s_{2} s_{1} n_{3}$ \\
-1.89736 & $n x_{3}$ & -0.04646 & $x_{3}^{2} s_{3}$ & -0.16394 & $s_{1}^{2} n_{3}$ \\
-2.30240 & $n s_{3}$ & +0.32321 & $x_{3}^{2} n_{3}$ & +0.04978 & $x_{3}^{3}$ \\
-2.18924 & $n n_{3}$ & +0.53257 & $s_{3}^{2} n_{3}$ & +0.67300 & $n s_{1}^{2}$ \\
-0.29380 & $x_{3}^{2}$ & +0.47298 & $s_{3} n_{3}^{2}$ & +1.09409 & $s_{1}^{2} s_{3}$ \\
-1.04511 & $x_{3} s_{3}$ & -0.04983 & $x_{3} s_{3} n$ & -0.06681 & $s_{2}^{2} n_{3}$ \\
+5.12780 & $x_{3} n_{3}$ & -0.02121 & $s_{3}^{3}$ & +0.72853 & $x_{3} s_{3} n_{3}$ \\
-0.94496 & $s_{3}^{2}$ & -0.46372 & $s_{1} s_{2} s_{3}$ & +0.63782 & $x_{3} n n_{3}$ \\
-2.31425 & $s_{3} n_{3}$ & +0.06086 & $n^{2} s_{3}$ & +0.15188 & $x_{3} s_{1}^{2}$ \\
-5.93052 & $n_{3}^{2}$ & -0.06517 & $n s_{3}^{2}$ & -1.08630 & $n^{2} n_{3}$ \\
-1.97105 & $s_{1}^{2}$ & +0.37455 & $s_{2} x_{3} s_{1}$ & +1.29031 & $n n_{3}^{2}$ \\
+7.47017 & $s_{1} s_{2}$ & -0.05827 & $x_{3} n^{2}$ & -0.61914 & $s_{3} n n_{3}$ \\
-13.03191 & $s_{2}^{2}$ & +0.10697 & $x_{3} s_{3}^{2}$ & -0.02940 & $n s_{1} s_{2}$ \\
& & -0.16039 & $x_{3} n_{3}^{2}$ & -0.07270 & $n s_{2}^{2}$ \\
& & +0.04477 & $n^{3}$ & -0.71215 & $s_{2}^{2} s_{3}$ \\
& & & & -0.18590 & $s_{2}^{2} x_{3}$ \\
\hline
\end{tabular}

\subsection{Reduced Hamiltonian}

We normalize the initial Taylor series expanded Hamiltonian to order $\epsilon^{4}$ (degree 6 in $z$, see Table 1) using standard Lie series technique [8a-d]. The transformed Hamiltonian, or the first normal form

$\mathcal{H}_{n}=923.488 n+208.872 x_{3}+163.455 n_{3}+\cdots$

can be expressed in terms of invariants as shown in Table 3 . We now fix the value of $n$ and consider it as a parameter. This defines the first reduced Hamiltonian $\mathcal{H}_{n}$ as a function on the first reduced phase space $P_{n} \sim \mathbb{C} P_{n}^{2}$.

\subsection{Stationary points of $\mathcal{H}_{n}$}

Qualitative analysis of possible stationary points of the reduced system with Hamiltonian $\mathcal{H}_{n}$ begins with the premise that $\mathcal{H}_{n}$ is a $C_{2} \times \mathcal{T}$ invariant Morse function on the reduced phase space $\mathbb{C} P^{2}$. Appendices $\mathrm{B}$ and $\mathrm{C}$ (see in particular Table B.1 and Fig. B.1) explain how symmetry and topology arguments can be used to find stationary points of such function. In particular we show that the asymmetric stretch RE (as) has fixed coordinates on $\mathbb{C} P^{2}{ }_{n}$ while symmetric stretch (ss) and bending (b) RE lie on the $C_{2} \times \mathcal{T}$ invariant circle $\mathbb{S}^{1}$.

When energies are close to 0 , the ss and $b$ points correspond to the symmetric stretch and bending normal modes. The two modes have the same symmetry and mix when $n$ increases. Position of ss and $\mathrm{b}$ on $\mathbb{C} P_{n}^{2}$ depends on $n$, see Appendix $\mathrm{C}$ and Table 4. Using positions of the three RE we compute their energy-action characteristics $E(n)$ as the value of $\mathcal{H}_{n}$ (Table 3 ) at the corresponding points on $\mathbb{C} P_{n}^{2}$, and com-
Table 4

Relative equilibria of ozone on the reduced phase space $\mathbb{C} P_{n}^{2}$ : values of invariants (Table A.1 in Appendix A) and energy obtained from the first normal form $\mathcal{H}_{n}$, which was computed to order 4

\begin{tabular}{llll}
\hline $\mathrm{RE}$ & $n_{3}$ & $s_{3}$ & $x_{3}$ \\
\hline ss & 0 & $-1.6026 \times 10^{-2} n^{2}$ & $n-1.2842 \times 10^{-4} n^{3}$ \\
& & $-2.1529 \times 10^{-4} n^{3}$ & 0 \\
as & $n$ & 0 & $-n+1.8116 \times 10^{-5} n^{3}$ \\
b & 0 & $6.0194 \times 10^{-3} n^{2}$ & Value of $\mathcal{H}_{n}($ energy) \\
RE & \multicolumn{3}{c}{$1132.3600 n-5.03156 n^{2}-1.68437 \times 10^{-3} n^{3}$} \\
ss & $1086.9425 n-10.96017 n^{2}+0.24877 n^{3}$ \\
as & \multicolumn{3}{c}{$714.6150 n-1.23684 n^{2}-1.53112 \times 10^{-2} n^{3}$} \\
b & \multicolumn{3}{c}{}
\end{tabular}

For all RE $s_{1}=s_{2}=t_{1}=t_{2}=t_{3}=0$.

pare this characteristics to numerically computed actions in Fig. 3.

It should be noted that errors in the normal form expressions for $E(n)$ at $n \approx 10$ are of the same order as (or superior to) the contribution due to the $n^{3}$ term in $E(n)$. This inaccuracy of the high order normal form can (at least partly) be attributed to the globally inaccurate representation of the initial Hamiltonian in terms of Taylor series near the equilibrium, see Section 6.

\subsection{Stretching polyads of ozone with resonance 1:1}

Like in many triatomic molecules, the frequencies of symmetric and antisymmetric stretching modes of ozone, $\omega_{1}$ and $\omega_{3}$ are very close to a 1:1 resonance and are known to form polyads. In order to describe these stretching polyads within our approach we introduce the second additional approximate integral of motion (additional dynamical symmetry) $n_{s}=n_{1}+n_{3}$ and normalize again as explained in

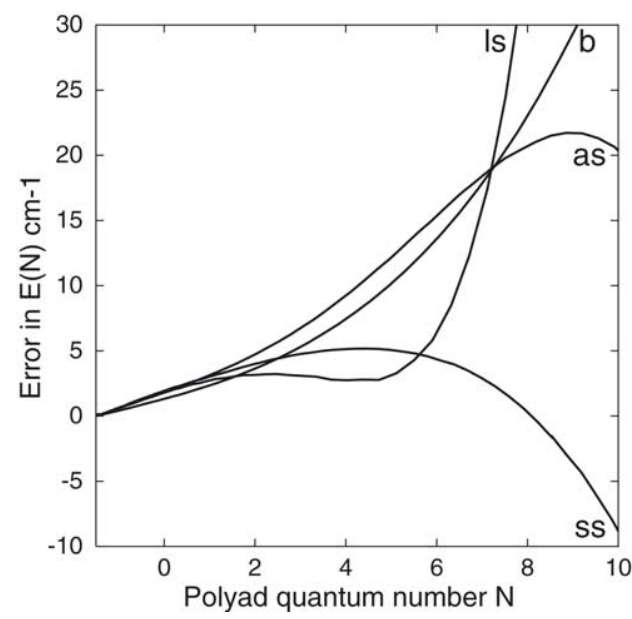

Fig. 3. Comparison of the analytical formulas for the energy-action characteristics of RE obtained from the normal form $\mathcal{H}_{n}$ and computed numerically for periodic orbits; ss, as, b, and ls denote symmetric stretch, asymmetric stretch, bending, and local stretch RE. 
Table 5

Terms in the second normalized Hamiltonian $\mathcal{H}_{n, n_{s}}$ describing 1:1 stretching polyads of ozone

\begin{tabular}{|c|c|c|c|c|c|}
\hline \multicolumn{2}{|l|}{$H_{n, n_{s}}^{(0)}$} & \multicolumn{2}{|l|}{$\epsilon^{2} H_{n, n_{s}}^{(1)}$} & \multicolumn{2}{|l|}{$\epsilon^{4} H_{n, n_{s}}^{(2)}$} \\
\hline 714.615 & $n$ & -1.2368 & $n^{2}$ & -0.015311 & $n^{3}$ \\
\hline 395.036 & $n_{s}$ & -8.5391 & $n n_{s}$ & -0.027586 & $n^{2} n_{s}$ \\
\hline-22.708 & $x_{2}$ & +4.6181 & $n_{s}^{2}$ & +0.114265 & $n n_{s}^{2}$ \\
\hline & & +1.0654 & $n_{s} x_{2}$ & -0.082545 & $n_{s}^{3}$ \\
\hline & & -4.0297 & $n x_{2}$ & +0.197438 & $n n_{s} x_{2}$ \\
\hline & & -2.8380 & $x_{2}^{2}$ & -0.102746 & $n^{2} x_{2}$ \\
\hline & & -13.0319 & $s_{2}^{2}$ & -0.022418 & $n_{s}^{2} x_{2}$ \\
\hline & & & & +0.017961 & $n x_{2}^{2}$ \\
\hline & & & & +0.116763 & $n_{s} x_{2}^{2}$ \\
\hline & & & & -0.576685 & $n$ \\
\hline & & & & +0.372594 & $n_{s} s_{2}^{2}$ \\
\hline & & & & +0.122691 & $x_{2} s_{2}^{2}$ \\
\hline & & & & +0.052958 & $x_{2}^{3}$ \\
\hline
\end{tabular}

Appendix D. To order $\epsilon^{4}$ the second reduced Hamiltonian

$\mathcal{H}_{n, n_{s}}=\mathcal{H}_{n, n_{s}}^{(0)}+\mathcal{H}_{n, n_{s}}^{(1)}+\mathcal{H}_{n, n_{s}}^{(2)}+\cdots$,

is given in Table 5. Notice that $0 \leq n_{s} \leq n$. It is also convenient to use the complimentary quantity $\delta=n-n_{s}$ (the "bending mode" action), such that $n \geq \delta \geq 0$.

Analysis of the second reduced system is entirely analogous to that of the rotational energy surfaces [6] and effective Hamiltonians on the polyad sphere [12] (see Section 1). We look for equilibria (stationary points) of the Hamiltonian function $\mathcal{H}_{n, n_{s}}$ defined on the 2-sphere $\mathbb{S}_{n_{s}}^{2}$. In the stretching and bending limit with $n_{s}=n(\delta=0)$ and $n_{s}=0(\delta=n)$, respectively, these equilibria lift to periodic orbits in the phase space $\mathbb{R}_{(q, p)}^{6}$ of the original system; when $0<n_{s}<n$ they lift to 2-tori. The former are, of course, relative equilibria (RE) which we already studied in Section 4.2. The latter can be also qualified as RE's in a broader sense (see Appendix A.5). The limiting "purely" stretching polyad with $n_{s}=n$ is at the top hyperpolyad energy and is represented by the energies of the three stretching RE, the symmetric, asymmetric, and local stretching modes, shown in Figs. 1 and 2.

Relative equilibria of $\mathcal{H}_{n, n_{s}}$ are analyzed in Appendix D.3. The two fixed RE correspond to the critical orbits of the $C_{2} \times$ $\mathcal{T}$ action on the second reduced phase space $\mathbb{S}_{n_{s}}^{2}$ illustrated in Fig. 4, left. Their energy $H_{n, n_{s}}$ is given in Table 6. In the limit of $n_{s}=n$ and $n_{s}=0$ expressions in this table equal those for stretching and bending RE in Table 4. Local modes bifurcate from the (as) point at a very small $n_{\text {crit }}$, they remain $\mathcal{T}$-invariant and move on the $\mathcal{T}$-invariant circle as shown in Fig. 4. Position of these RE on $\mathbb{S}^{2}$ and the value of $\mathcal{H}_{n, n_{s}}$ is given in Table 7. Graphic representation of $\mathcal{H}_{n, n_{s}}$ in the region $n>n_{\text {crit }}$ is shown in Fig. 4, right.

\subsection{Classification of quantum states based on second normalization}

To compare our results for the relative equilibria of the second normalized system with the known quantum energy

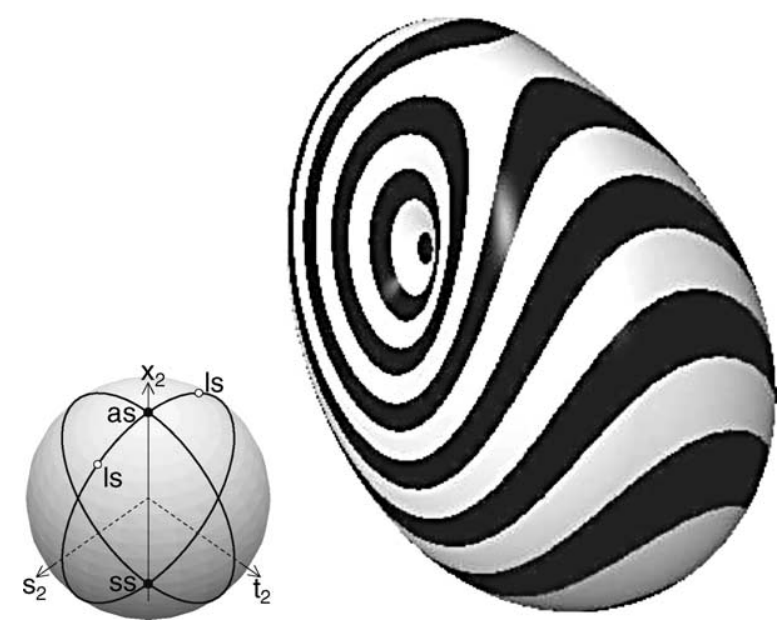

Fig. 4. Stratification of the second reduced phase space $\mathbb{S}^{2}$ (polyad sphere) under the action of $C_{2} \times \mathcal{T}$ (left). Black dots mark fixed points which correspond to relative equilibria (ss) and (as), white dots show local mode RE. Second normal form $\mathcal{H}_{n, n_{s}}$ for the 1:1 stretching polyad of ozone with $n_{s}=n=6$ as a function on $\mathbb{S}^{2}$ (right). Black and white stripes represent constant level sets of $\mathcal{H}_{n, n_{s}}$ with arbitrarily fixed spacing.

Table 6

Energy of relative equilibria of ozone which correspond to fixed points of the $C_{2} \times \mathcal{T}$ symmetry group action on the second reduced phase space $\mathbb{S}_{n, n_{s}}^{2}$

\begin{tabular}{lcl}
\hline ss : $x_{2}=-n_{s}$ & as : $x_{2}=n_{s}$ & Term \\
\hline 1132.3600 & 1086.9425 & $n$ \\
-417.7450 & -372.3275 & $\delta$ \\
-5.03156 & -10.96017 & $n^{2}$ \\
+3.08000 & +6.87774 & $n \delta$ \\
+0.71471 & +2.84558 & $\delta^{2}$ \\
$-1.68437 \times 10^{-3}$ & 0.24877 & $n^{3}$ \\
$+4.42281 \times 10^{-2}$ & -0.72327 & $n^{2} \delta$ \\
$-5.41770 \times 10^{-2}$ & +0.52393 & $n \delta^{2}$ \\
$-3.67798 \times 10^{-3}$ & $-6.47577 \times 10^{-2}$ & $\delta^{3}$ \\
\hline
\end{tabular}

The energy is obtained as the value of the second normal form $\mathcal{H}_{n, n_{s}}$ computed to order 4 ; note that $s_{2}=t_{2}=0$ and $\delta=n-n_{s} \geq 0$.

Table 7

Local mode stretching relative equilibrium of ozone: critical value $n_{\text {crit }}(\delta)$ of $n$ for the local mode bifurcation, coordinate $x_{2}(x, \delta)$, which defines the position of $1 \mathrm{~s}$ on the second reduced phase space $\mathbb{S}_{n, n_{s}}^{2}$, and energy $E^{\mathrm{ls}}(x, \delta)$ obtained from the second normal form $\mathcal{H}_{n, n_{s}}$

\begin{tabular}{llrl}
\hline$n_{\text {crit }}$ & \multicolumn{1}{l}{$x_{2}$} & \multicolumn{1}{l}{ Energy } & Term \\
\hline 1.24438 & 1.24438 & 1336.08580 & \\
1.13419 & 0.13419 & 838.29596 & $\delta$ \\
& $0.78983 \times 10^{-1}$ & 1060.83409 & $x$ \\
& $-1.19399 \times 10^{-2}$ & -18.79975 & $x^{2}$ \\
$-3.56332 \times 10^{-3}$ & $-1.12134 \times 10^{-2}$ & -17.66161 & $x \delta$ \\
& $-3.56332 \times 10^{-3}$ & -7.42360 & $\delta^{2}$ \\
& $3.67745 \times 10^{-4}$ & -0.18968 & $x^{3}$ \\
$2.40667 \times 10^{-4}$ & $1.03450 \times 10^{-3}$ & -0.59742 & $x^{2} \delta$ \\
& $8.20619 \times 10^{-4}$ & -0.07857 & $x \delta^{2}$ \\
\hline
\end{tabular}

Solutions are given in terms of powers of $x=n-n_{\text {crit }}>0$ and $\delta=n-$ $n_{s} \geq 0$ in the last column, e.g., $n_{\text {crit }}=1.24438+1.13419 \delta+\cdots$. 
spectrum of ozone [15a] we should introduce quantum number $N_{s}$ of the stretching 1:1 polyads. The combined quantumclassical correspondence rule is

$n=N+\frac{3}{2}, \quad N=0,1, \ldots$,

$n_{s}=N_{s}+1, \quad N_{s}=0,1, \ldots, N$.

Here the value of the classical action $n_{s}=n-\frac{1}{2}$ corresponds to the maximum quantum number $N_{s}$ in the hyperpolyad $N$; the half-quantum remains in the bending mode, $\mathrm{cf}$. Appendix E.

The energy-action plot of RE of the second normalized system is shown in Fig. 5. In the limiting cases $\left(n_{s}=n\right.$ for the stretching RE ss, as, and ls, and $n_{s}=0$ for the bending $\mathrm{RE}$ ) our curves represent the RE of the first normalized system shown in Figs. 1 and 2. When $n>n_{s}>0$, they represent the energy $\mathcal{H}_{n, n_{s}}$ of the RE of the second normalized system. The curves, representing such RE for the special values $\frac{1}{2}, 1 \frac{1}{2}$, and $2 \frac{1}{2}$ of the difference $n-n_{s}$, are plotted in Fig. 5. They correspond to the uppermost, second top, and third top quantum 1:1 polyad. The structure of these "small" polyads replicates the structure of the $n=n_{s}$ limit. While the 1:1 polyads overlap partially in energy, the RE of the same type remain well separated and the 1:1 approximation remains valid in the studied action range. However, the visualization of the corresponding quantum level structure is compromised. Despite the overlap and the incompleteness of the list of observed and assigned quantum levels of ozone provided in [15a,b], several doublets of quasidegenerate quantum levels corresponding to two equivalent RE of the local stretching mode ls can be seen near the bottom of 1:1 polyads. A more detailed analysis is possible using quantum energies in Table 8 in the next section.

\subsection{Quantization of the second normal form}

We quantize our second normal form $\mathcal{H}_{n, n_{s}}$ in order to detail the global qualitative description of the whole energy spectrum. As shown in Appendix D.3, invariants $\left(\frac{1}{2} x_{2}, \frac{1}{2} s_{2}, \frac{1}{2} t_{2}\right)$ generate a Poisson algebra which is a standard so(3) with Casimir $j=\frac{1}{2} n_{s}$. We replace these invariants by angular momentum operators $\left(\hat{\jmath}_{1}, \hat{\jmath}_{2}, \hat{\jmath}_{3}\right)$ and diagonalize the $(2 j+1) \times(2 j+1)$ matrix of $\hat{H}_{n, n_{s}}\left(\hat{\jmath}_{1}, \hat{\jmath}_{2}, \hat{\jmath}_{3}\right)$ in the standard basis of spherical harmonic functions $|j, m\rangle$.

Of course, we should not forget the obvious limitations of our classical normalization and the above quantization. Both remain correct only in the sense of main (=classical) contributions to the high degree terms. Thus starting with the classical function $\mathcal{H}_{n, n_{\mathrm{s}}}$, we have no way to define correct ordering of noncommuting operators $\left(\hat{\jmath}_{1}, \hat{\jmath}_{2}, \hat{\jmath}_{3}\right)$. The best we can do is to use symmetrized Hermitian combinations.

Despite all apprehension, our method provides excellent results shown in Table 8. Increasing errors for higher polyads should be explained (at least partially) not by the breakdown of the polyad approximation but rather by the deficiency of the Taylor series representation of the initial Hamiltonian.

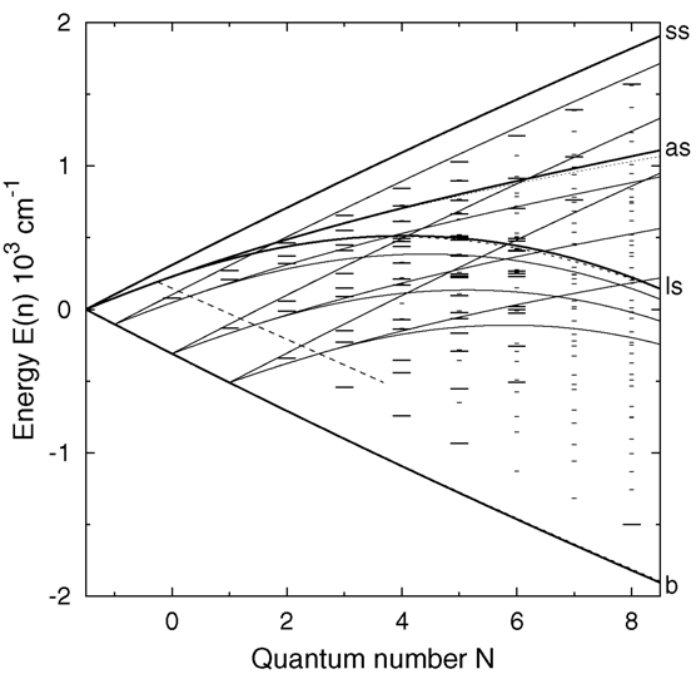

Fig. 5. Energy of relative equilibria, the polyad structure, and quantum levels of ozone. Bold solid lines show the energy of stationary points of $\mathcal{H}_{n}$; fine dotted lines show corresponding numeric energy-action data (in the scale of the figure they can only be seen for as and ls RE at high energy); fine solid lines show the energy of stationary points of $\mathcal{H}_{n, n_{s}}$ with $n-n_{s}$ equal to $\frac{1}{2}, 1 \frac{1}{2}$, and $2 \frac{1}{2}$; the dashed line marks the point of the as $\rightarrow$ ls bifurcation. Short and long ticks show levels assigned in $[15 \mathrm{a}, \mathrm{b}]$ and computed in Section 4.5 , respectively. The base quantity $1378.15+914.027 N-3.1724 N^{2}-0.0084978 N^{3}$ is subtracted.

\section{Table 8}

Energies $E$ of vibrational quantum states of ozone computed using the quantized second normal form $\mathcal{H}_{n, n_{s}}$ with subtracted zero point energy of 1455.3

\begin{tabular}{lrrrrrrrlllll}
\hline \multicolumn{1}{l}{ State } & \multicolumn{1}{l}{$E\left(\mathrm{~cm}^{-1}\right)$} & \multicolumn{2}{l}{ State } & \multicolumn{2}{l}{$E\left(\mathrm{~cm}^{-1}\right)$} & State & \multicolumn{3}{l}{$E\left(\mathrm{~cm}^{-1}\right)$} \\
\hline 00 & 000 & 1 & 1 & 43 & 211 & 3855 & 6 & 60 & & 4163 & \\
10 & 010 & 702 & 1 & 43 & 310 & 3968 & 1 & 61 & & 4436 & \\
11 & 001 & 1043 & 1 & 44 & 004 & 4006 & 5 & 61 & & 4543 & \\
11 & 100 & 1103 & 0 & 44 & 103 & 4028 & 6 & 62 & & 4715 & \\
20 & 020 & 1400 & 1 & 44 & 202 & 4146 & 5 & 62 & 141 & 4799 & 15 \\
21 & 011 & 1728 & 1 & 44 & 301 & 4253 & 3 & 62 & & 4928 & \\
21 & 110 & 1797 & 0 & 44 & 400 & 4368 & -2 & 63 & & 4998 & \\
22 & 002 & 2059 & 1 & 50 & 050 & 3477 & -1 & 63 & 132 & 5055 & 19 \\
22 & 101 & 2112 & 1 & 51 & & 3764 & & 63 & & 5181 & \\
22 & 200 & 2201 & 0 & 51 & 140 & 3861 & 3 & 64 & 024 & 5280 & 14 \\
30 & 030 & 2095 & 0 & 52 & & 4057 & & 64 & 123 & 5309 & 18 \\
31 & 021 & 2410 & 2 & 52 & 131 & 4132 & 10 & 63 & 330 & 5317 & 7 \\
31 & 120 & 2488 & 1 & 52 & 230 & 4251 & 4 & 64 & & 5441 & \\
32 & 012 & 2728 & 2 & 53 & 023 & 4352 & 6 & 65 & 015 & 5548 & 29 \\
32 & 111 & 2788 & 3 & 53 & 122 & 4402 & 12 & 65 & 114 & 5558 & 17 \\
32 & 210 & 2887 & 0 & 53 & 221 & 4520 & 12 & 64 & 321 & 5568 & 10 \\
33 & 003 & 3048 & 2 & 53 & 320 & 4644 & 0 & 64 & 420 & 5708 & 6 \\
33 & 102 & 3086 & 3 & 54 & 014 & 4645 & 12 & 65 & 213 & 5716 & 18 \\
33 & 201 & 3188 & 2 & 54 & 113 & 4670 & 11 & 66 & 006 & 5796 & 29 \\
33 & 300 & 3289 & -1 & 54 & 212 & 4795 & 12 & 66 & 105 & 5798 & 14 \\
40 & 040 & 2788 & 0 & 54 & 311 & 4912 & 15 & 65 & & 5820 & \\
41 & 031 & 3088 & 2 & 55 & 005 & 4924 & 5 & 65 & & 5962 & \\
41 & 130 & 3176 & 2 & 55 & 104 & 4932 & 10 & 66 & 204 & 6010 & 15 \\
42 & 022 & 3394 & 3 & 54 & & 5039 & & 66 & & 6077 & \\
42 & 121 & 3462 & 6 & 55 & 203 & 5086 & 9 & 65 & & 6102 & \\
42 & & 3570 & & 55 & 302 & 5180 & 9 & 66 & 402 & 6217 & 12 \\
43 & 013 & 3702 & 4 & 55 & 401 & 5312 & 4 & 66 & & 6361 & \\
43 & 112 & 3746 & 6 & 55 & 500 & 5438 & -3 & 66 & 600 & 6498 & -4 \\
\hline
\end{tabular}

Each state is characterized by quantum numbers $N$ and $N_{s}$. For states listed in [15a], the normal mode assignment $\left[v_{1} v_{2} v_{3}\right]$ (second column) and the $E-E_{\mathrm{obs}}$ difference (last column) are given. 
Note that similar factors cause the divergence of classical RE energies illustrated in Fig. 3.

\section{Direct polyad assignment}

To demonstrate the validity and utility of our hyperpolyad classification, we took quantum energy levels of ozone identified in [15a,b]. However, the normal (or local) mode identification of computed quantum levels often turns out difficult and even prohibitive. As pointed to us by Tyuterev [22], one of the authors of the direct quantum computation for ozone [15a,b], assignment in terms of conventional normal-mode quantum numbers $\left(v_{1}, v_{2}, v_{3}\right)$ becomes increasingly dubious starting with $N=4, \ldots, 6$. In this region, wavefunctions become heavy mixtures distributed over many basis functions. Often there is no clearly dominant function and sometimes the leading contribution can be as low as 5\%. Furthermore, the node analysis often does not confirm the traditional "spectroscopic" assignment. This all is further complicated by the problem of internal-to-normal coordinate conversion and by the extreme sensitivity of some of the predicted states to small variations of potential parameters and basis. It should be pointed out, that similar difficulties are typical for other molecules.

We saw that the polyad numbers $N$ and $N_{S}$ of ozone remained valid at high excitations, while the individual normal mode assignments $\left(v_{1}, v_{2}, v_{3}\right)$ were no longer applicable. In principle, $N$ (and $N_{s}$ ) can be computed directly from quantum wavefunctions. Since wavefunctions are defined on the original configuration space, we should first express $n$ in terms of the original normal mode coordinates $q$ and conjugate momenta $p$, see Appendix A. This gives a series

$\mathcal{L}^{-1} n=n+\epsilon\left[\mathcal{L}^{-1} n\right]_{1}+\frac{1}{2} \epsilon^{2}\left[\mathcal{L}^{-1} n\right]_{2}+\cdots$,

whose high order terms $\left[\mathcal{L}^{-1} n\right]_{k}$ are given in Table 9 . The polyad number $N$ is the expectation value $\left\langle\mathcal{L}^{-1} n\right\rangle$ for the quantum analogue of $\mathcal{L}^{-1} n$.

In order to estimate the accuracy of the proposed method, we checked how well the quantity $\mathcal{L}^{-1} n$ in (5) (truncated to some order) is conserved along the trajectories of the original system in the phase space $\mathbb{R}_{(q, p)}^{6}$. This could be done easily for the RE trajectories found in Section 3. At a given fixed value of action and energy, we computed the action $\int p \mathrm{~d} q$ and $2 \pi \mathcal{L}^{-1} n$ along RE and studied their difference. In most cases this difference remained small; in some cases, however, it peaked at sharp turning points, where our Taylor series reproduced the potential particularly poorly.

We should admit that in practice, the proposed assignment method has a number of limitations. For example, the $\mathcal{L}^{-1} n$ quantity is obtained as a series in normal mode coordinates $(q, p)$. Its extrapolation to high energies can be made difficult not only due to the "legitimate" restrictions of the polyad approximation, but also because the Taylor series fails to re-
Table 9

Hyperpolyad invariant for the potential in [15a] expressed using initial normal mode coordinates

\begin{tabular}{|c|c|c|c|c|}
\hline Order & Coefficient & Term & Coefficient & Term \\
\hline$\epsilon^{0}$ & 1 & $n$ & & \\
\hline \multirow[t]{8}{*}{$\epsilon^{1} \times 10^{-2}$} & -4.53769 & $\bar{z}_{1} \bar{z}_{3} z_{3}$ & -0.72735 & $\bar{z}_{1} \bar{z}_{2} z_{2}$ \\
\hline & 1.83110 & $\bar{z}_{2} \bar{z}_{3} z_{3}$ & 1.02949 & $\bar{z}_{1} z_{1} \bar{z}_{2}$ \\
\hline & -0.42743 & $z_{1}^{2} \bar{z}_{2}$ & -2.15952 & $z_{1} \bar{z}_{3}^{2}$ \\
\hline & -2.15393 & $\bar{z}_{1} \bar{z}_{3}^{2}$ & 0.79289 & $z_{2} \bar{z}_{3}^{2}$ \\
\hline & 0.17609 & $z_{1} \bar{z}_{2}^{2}$ & 0.40596 & $z_{1} z_{2}^{2}$ \\
\hline & 0.85740 & $z_{2} z_{3}^{2}$ & 1.45156 & $\bar{z}_{2} z_{2}^{2}$ \\
\hline & 0.37588 & $\bar{z}_{1}^{2} \bar{z}_{2}$ & -1.26316 & $\bar{z}_{1} z_{1}^{2}$ \\
\hline & 0.60643 & $\bar{z}_{2}^{3}$ & -0.51476 & $\bar{z}_{1}^{3}$ \\
\hline \multirow[t]{19}{*}{$\frac{1}{2} \epsilon^{2} \times 10^{-2}$} & 2.06150 & $\bar{z}_{1} z_{1} \bar{z}_{3} z_{3}$ & 0.436622 & $\bar{z}_{1} z_{1} \bar{z}_{2} z_{2}$ \\
\hline & 0.829110 & $\bar{z}_{2} z_{2} \bar{z}_{3} z_{3}$ & 0.260237 & $\bar{z}_{1}^{2} z_{1}^{2}$ \\
\hline & 0.319093 & $\bar{z}_{2}^{2} z_{2}^{2}$ & 0.817718 & $\bar{z}_{3}^{2} z_{3}^{2}$ \\
\hline & -0.362839 & $z_{1} z_{2} \bar{z}_{3} z_{3}$ & -0.431633 & $\bar{z}_{3} z_{3} \bar{z}_{1} z_{2}$ \\
\hline & 0.265534 & $\bar{z}_{1} z_{1} \bar{z}_{3}^{2}$ & -0.073159 & $\bar{z}_{1} z_{1} \bar{z}_{2}^{2}$ \\
\hline & 0.062587 & $\bar{z}_{2} z_{2} \bar{z}_{3}^{2}$ & -0.022140 & $\bar{z}_{1} \bar{z}_{2}^{2} z_{2}$ \\
\hline & -0.101320 & $\bar{z}_{2}^{2} \bar{z}_{3} z_{3}$ & -0.178183 & $\bar{z}_{1} z_{1}^{2} z_{2}$ \\
\hline & 0.040851 & $z_{1}^{2} \bar{z}_{2} z_{2}$ & 0.307898 & $z_{1}^{2} \bar{z}_{3} z_{3}$ \\
\hline & -0.207081 & $z_{1} z_{2} z_{3}^{2}$ & -0.192657 & $z_{1} \bar{z}_{2} z_{3}^{2}$ \\
\hline & -0.188263 & $\bar{z}_{1} z_{2} z_{3}^{2}$ & -0.004925 & $\bar{z}_{1}^{2} z_{1} z_{2}$ \\
\hline & 0.010098 & $\bar{z}_{2}^{2} z_{2} z_{1}$ & -0.504487 & $z_{2} \bar{z}_{3}^{2} z_{1}$ \\
\hline & 0.001610 & $z_{1}^{4}$ & 0.032320 & $z_{2}^{4}$ \\
\hline & 0.049643 & $z_{3}^{4}$ & 0.018965 & $\bar{z}_{1}^{3} z_{1}$ \\
\hline & 0.198837 & $\bar{z}_{3}^{3} z_{3}$ & -0.052649 & $z_{1}^{3} z_{2}$ \\
\hline & -0.011783 & $z_{1} z_{2}^{3}$ & 0.154109 & $\bar{z}_{2} z_{2}^{3}$ \\
\hline & -0.061883 & $z_{1} \bar{z}_{2}^{3}$ & -0.033397 & $z_{1}^{3} \bar{z}_{2}$ \\
\hline & 0.141450 & $z_{1}^{2} z_{3}^{2}$ & -0.028745 & $z_{2}^{2} z_{3}^{2}$ \\
\hline & 0.008507 & $z_{1}^{2} z_{2}^{2}$ & 0.094462 & $\bar{z}_{2}^{2} z_{3}^{2}$ \\
\hline & 0.737032 & $\bar{z}_{1}^{2} z_{3}^{2}$ & -0.010975 & $\bar{z}_{1}^{2} z_{2}^{2}$ \\
\hline
\end{tabular}

Coordinates $(z, \bar{z})$ are defined in Appendix A; not self-conjugate monomials enter with their complex conjugate.

produce well the original Hamiltonian. On the quantum side, computing expectation values of high powers of momenta $p$ requires high order derivatives, which can be difficult to evaluate numerically. We believe, however, that in the case of ozone, using a few first orders of $\mathcal{L}^{-1} n$ should enable advancing hyperpolyad assignments well beyond the today's limit indicated in Fig. 5 and Table 8.

\section{Discussion}

In general, due to their rotator analogy, the two-mode polyads (such as the stretching polyads of ozone) are the only polyads, whose dynamical description is relatively widely implemented (see series of papers by Kellman and coworkers, such as $[12,23,14])$. Polyads formed by a larger 
number of modes are often introduced formally in relation to good quantum numbers (cf. $[13,24 d, f]$ ), and very few studies attempted to analyze them dynamically. Relative equilibria of such systems are commonly treated as simple periodic orbits, though their relation to the polyad integral of the normal form approximation and their role in understanding the internal structure of polyads is intuitively recognized [27].

Arguably, the principal obstacle to the dynamical analysis of polyads formed by a larger number of modes is the geometry of their reduced phase spaces. Thus, even though the $\mathbb{C} P^{2}$ polyad space of the 1:1:1 resonance system is sufficiently simple [31,32,33a-d] for being used as widely as the "polyad sphere", it is rarely mentioned in molecular literature on three mode systems. It is possible that some researchers avoid $\mathbb{C} P^{2}$ because they underestimate the broadness of the 1:1:1 resonance in an anharmonic system and favor more elaborate resonance models, which reproduce more closely the ratio of the harmonic frequencies of the molecule, e.g. 5:3:5 in the case of ozone. We hope that our present work would finally convince most of the skeptics of the versatility of $\mathbb{C} P^{2}$

It should be noted that resonances complicate the situation even further. Thus, in the simplest case of the two mode oscillator system with higher resonances, the reduced phase space is a topological sphere, which is, unlike the 1:1 polyad space, not smooth. It has been discussed comprehensively in the mathematical literature in the example of the "classic" two-mode Fermi system, or the 1:2 resonance; see Fig. 4.1 of Appendix B.4 (Example 3) in [34,36, p. 40], and the semiclassical analysis in [35]. Regrettably, this mathematical work has been as yet of little impact on the molecular community, and the geometry of the $m_{1}: m_{2}$ polyad spaces is still not clearly understood [37]. Geometry of the three mode polyad spaces in the case of higher resonances can be very complex. Such spaces are known as weighted projective spaces [38]. Only in some cases, such as 1:2:2 used in [14] to model ozone, they can be described as $\mathbb{C} P^{2}$ with singularities. The best known counterexample is the 1:1:2 polyad space, whose geometry is very difficult to trivialize [39]. However, this geometry can be simplified due to an additional Lie symmetry, such as the axial $\mathrm{SO}(2)$ symmetry of linear triatomic molecules. The $\mathrm{CO}_{2}$ molecule and its mechanical analogues is the most recently revisited interesting example [40a-c], where singular reduction of both the 1:1:2 polyad symmetry and the $S O(2)$ symmetry results in an integrable approximation with two respective first integrals $n \geq 0$ and $-n<\ell<n$ and the reduced phase space, which is either a topological sphere (for $\ell=0$ ) or a smooth sphere $\mathbb{S}^{2}$. Our 1:1:1 model of ozone, where we also introduce the second Lie symmetry, is simpler because reduction is regular and all second reduced phase spaces with $n_{s}<n$ are the same (Section 4.3).

The above observations led to our two principal motivations for this paper: (i) to uncover once again the dynamics and phase space geometry of higher polyad systems in a fun- damental molecular system of current interest, and (ii) to make explicit the relationship between the polyad approximation and RE. Far from trying to be didactic, we like to complement the significant body of the contemporary work on the vibrational analysis of triatomic and polyatomic molecules. We strongly believe that only complete understanding of the hyperpolyad dynamics can enable further progress in this field.

Figs. 2 and 5 illustrate the central role, which RE play in shaping the hyperpolyad structure of the quantum energy level spectrum. This relation of RE to the existence of hyperpolyads is further confirmed by the agreement of numerical action-energy data (Section 3) with the analytical expansions for $E(n)$ obtained in the hyperpolyad approximation (Table 4). In fact, the deviation of the two is almost invisible on the scale of Fig. 2.

It is tempting to explain, why this works so well in the case of ozone and to generalize, if possible, beyond this case. The answer is, however, difficult to give quantitatively. The most important resonance in ozone is the $1: 1$ resonance of stretching modes. This resonance is included in the 1:1:1 polyad model and is treated correctly. At the same time, the bending-to-stretching frequency ratio of 3:5 is sufficiently far from the Fermi resonance 1:2 and can be ignored at low polyad numbers $n$. We can, therefore, argue that the plain 1:1:1 hyperpolyad approximation applies in cases which are far from exact low-order resonances. Furthermore, the lowest 3:5 resonance term is of degree 8 , while our present study is limited to degree 6 (order $\epsilon^{4}$ ). Indeed, Fig. 3 suggests that our normal form formulas diverge quickly when $n$ approaches $8, \ldots, 10$. At the same time, another, more obvious source of this divergence is the inefficiency of the Taylor series approximation of the potential [15a], which goes bad at degrees higher than 4 .

It is indisputable and, in fact, widely accepted, that the RE-based analysis is a basic and very effective tool, which can be used in systems with many degrees of freedom. It will be interesting to study how it can be extended to relative periodic orbits (Appendix A.5) and find applications of such extension in real molecular systems. Another very promising generalization is the analysis based on rotationvibration relative equilibria [33d]. It will be equally interesting to study continuation of RE to high energies (cf. [29]) in order to understand the breakdown of the polyad approximation.

\section{Acknowledgments}

This work was supported by the EU project Mechanics and Symmetry in Europe (MASIE), contract HPRN-CT-200000113. We thank Dr. M. Roberts for his help with continuation of periodic orbits, and Dr. M. Joyeux for his comments. We thank Dr. Vl.G. Tyuterev for providing us with his data and potential [15a] prior to their publication and his comments. 


\section{Appendix A. Reduction of the 1:1:1 symmetry and first normal form}

Consider the vector field $X_{n}$ of the 1:1:1 harmonic oscillator with Hamiltonian

$n=\frac{1}{2}\left(q_{1}^{2}+p_{1}^{2}+q_{2}^{2}+p_{2}^{2}+q_{3}^{2}+p_{3}^{2}\right)$.

In complex phase space coordinates

$z_{k}=q_{k}-\mathrm{i} p_{k}, \quad \bar{z}_{k}=q_{k}+\mathrm{i} p_{k}, \quad k=1,2,3$,

the flow of $X_{n}$ is a rotation

$\varphi_{n}:\left(z_{1}, z_{2}, z_{3}, t\right) \rightarrow\left(\mathrm{e}^{\mathrm{i} t} z_{1}, \mathrm{e}^{\mathrm{i} t} z_{2}, \mathrm{e}^{\mathrm{i} t} z_{3}\right)$

of the complex space $\mathbb{C}^{3} \sim \mathbb{R}_{(q, p)}^{6}$.

We transform the initial phase space $\mathbb{C}^{3}$ into the phase space $\tilde{\mathbb{C}}^{3}$ of the normalized system using a near identity transformation $\mathcal{L}$, which can be constructed as a Lie series [8a-d]. The transformed Hamiltonian $\mathcal{H}$, or the normal form, is a formal power series in variables $\tilde{z}$. We expect that in the normalized coordinates $\tilde{z}$ the Hamiltonian flow $\varphi_{H}$ of our initial system has $\varphi_{n}$ as a component. In other words, all terms in the series $\mathcal{H}$ Poisson commute with $n$, i.e., they are invariant with respect to the oscillator symmetry $\varphi_{n}$. We can, therefore, reduce the $\varphi_{n}$ component.

After the near identity normalization transformation $\mathcal{L}$ is constructed, we can find the inverse $\mathcal{L}^{-1}: \tilde{\mathbb{C}}^{3} \rightarrow \mathbb{C}^{3}$ also in the form of a Lie series [8a-d]. On the transformed space $\tilde{\mathbb{C}}^{3}$, the integral $n$ equals the quadratic form in (A.1), and RE are geometric circles $\mathbb{S}^{1}$. The $\mathcal{L}^{-1}$ transformation is necessary to reconstruct $n$ and its orbits RE for the original system. In particular, $n$ becomes a series $\mathcal{L}^{-1} n$ in $(z, \bar{z})$, see Eq. (5) and Table 9.

\section{A.1. Reduced phase space}

Since $\varphi_{n}$ acts freely on $\tilde{\mathbb{C}}^{3}$ (for all $n>0$ ) reduction is straightforward. We descend on the constant level set of $n$ (which is a hypersphere of radius $\rho=\sqrt{2 n}$ ) and identify all points of this set which belong to the same circular orbit of $\varphi_{n}$. Hence a point on the reduced phase space $P_{n}$, which we call the hyperpolyad space, corresponds to all points $\tilde{z}$ on the transformed phase space $\tilde{\mathbb{C}}^{3}$ which have the same module $|\tilde{z}|=\rho$ and which differ only in total phase exp(it). It follows that for any $n>0$ the space $P_{n}$ is isomorphic to a complex projective space $\mathbb{C} P^{2}$.

One possible etymology of the term hyperpolyad is that the associated polyad integral $n$ in (A.1) and the polyad number $n$ in (1) are related directly to the hyperradius $\varrho$ in the vibrational phase space of the system:

$n=\frac{1}{2} \sum\left(p_{i}^{2}+q_{i}^{2}\right)=\frac{1}{2} \varrho^{2}$.
Table A.1

Invariant polynomials of the 1:1:1 oscillator action used in the construction and analysis of the hyperpolyad approximation for a molecule with three vibrational modes

\begin{tabular}{llll}
\hline & Expression & & Expression \\
\hline$n$ & $\frac{1}{2}\left(z_{1} \bar{z}_{1}+z_{2} \bar{z}_{2}+z_{3} \bar{z}_{3}\right)$ & $n_{s}$ & $\frac{1}{2}\left(z_{1} \bar{z}_{1}+z_{3} \bar{z}_{3}\right)$ \\
$n_{1}$ & $\frac{1}{2} z_{1} \bar{z}_{1}$ & $x_{1}$ & $\frac{1}{2}\left(z_{2} \bar{z}_{2}-z_{3} \bar{z}_{3}\right)$ \\
$n_{2}$ & $\frac{1}{2} z_{2} \bar{z}_{2}$ & $x_{2}$ & $\frac{1}{2}\left(z_{3} \bar{z}_{3}-z_{1} \bar{z}_{1}\right)$ \\
$n_{3}$ & $\frac{1}{2} z_{3} \bar{z}_{3}$ & $x_{3}$ & $\frac{1}{2}\left(z_{1} \bar{z}_{1}-z_{2} \bar{z}_{2}\right)$ \\
$s_{1}$ & $\frac{1}{2}\left(z_{2} \bar{z}_{3}+z_{3} \bar{z}_{2}\right)$ & $t_{1}$ & $\frac{1}{2} \mathrm{i}\left(z_{2} \bar{z}_{3}-z_{3} \bar{z}_{2}\right)$ \\
$s_{2}$ & $\frac{1}{2}\left(z_{3} \bar{z}_{1}+z_{1} \bar{z}_{3}\right)$ & $t_{2}$ & $\frac{1}{2} \mathrm{i}\left(z_{3} \bar{z}_{1}-z_{1} \bar{z}_{3}\right)$ \\
$s_{3}$ & $\frac{1}{2}\left(z_{1} \bar{z}_{2}+z_{2} \bar{z}_{1}\right)$ & $t_{3}$ & $\frac{1}{2} \mathrm{i}\left(z_{1} \bar{z}_{2}-z_{2} \bar{z}_{1}\right)$ \\
\hline
\end{tabular}

At the same time, such terminology helps to distinguish hyperpolyads of ozone from the widely known stretching polyads of this molecule, where one hyperpolyad contains a family of "smaller" purely stretching polyads.

\section{A.2. Invariant polynomials}

Consider all polynomials in $(z, \bar{z})$ which are invariant with respect to the flow $\varphi_{n}$ of the vector field $X_{n}$ of the $1: 1: 1$ oscillator. In other words, consider all polynomials in $(z, \bar{z})$ which Poisson commute with $n$. A direct calculation shows that the total degrees in $\left(z_{1}, z_{2}, z_{3}\right)$ and $\left(\bar{z}_{1}, \bar{z}_{2}, \bar{z}_{3}\right)$ of these polynomials must equal. The multiplicative ring of all $\varphi_{n}$ invariant polynomials is generated by quadratic polynomials of the form $z \bar{z}$. Our particular choice of such generators is presented in Table A.1.

\section{A.3. Integrity basis}

Any $\varphi_{n}$-invariant polynomial function, such as the normal form $\mathcal{H}$, can be expressed in terms of invariants in Table A.1. Although these invariants are linearly independent, there is a number of algebraic dependencies (or "sygyzies") among them. This means that the expression of $\mathcal{H}$ may not be unique. We say that the ring of all $\varphi_{n}$-invariant polynomials is not generated freely.

It is possible, however, to represent $\mathcal{H}$ uniquely using the so-called integrity basis whose particular realization is

$\mathcal{R}\left(n ; x_{3}, s_{1}, s_{2}, s_{3}\right) \times\left\{1, n_{3}, n_{3}^{2}, t_{1}, t_{2}, t_{3}\right\}$.

Here the ring $\mathcal{R}$ is freely generated by the so-called principal invariants $\left(x_{3}, s_{1}, s_{2}, s_{3}\right)$ and $n$. (These invariants enter in arbitrary degrees.) All other invariants are auxiliary and should be used as factors of degree 1 or 0 .

\section{A.4. Reminder on the two mode case}

For a lower dimensional example consider a similar procedure for the 1:1 oscillator. In this case, the polyad space is the space $\mathbb{C} P^{1}$ which is diffeomorphic to a 2 -sphere $\mathbb{S}^{2}$ [9]. This space is often called the polyad phase sphere [12]. Generators $\left(j_{1}, j_{2}, j_{3}\right)$ of the ring of invariant polynomials are 
angular momentum components defined in the way similar to $\left(\frac{1}{2} x_{3}, \frac{1}{2} s_{3}, \frac{1}{2} t_{3}\right)$ and bound by the relation

$j^{2}=j_{1}^{2}+j_{2}^{2}+j_{3}^{2}$

where $2 j$ is, of course, equivalent to the polyad integral $n$. With no other symmetries (such as time reversal, etc) taken into account this ring has the structure

$\mathcal{R}\left(j ; j_{1}, j_{2}\right) \times\left\{1, j_{3}\right\}$

In this paper, we work with two different realizations of the above construction: the $C_{2}$-invariant $\mathbb{S}^{2}$ subspace of $\mathbb{C} P^{2}$ and the phase space of the second reduced problem in the approximation of the 1:1 stretching mode resonance. In the latter case, we use invariants $\left(x_{2}, s_{2}, t_{2}\right)$.

In quantum mechanics, an entirely analogous construction of angular momentum operators in terms of two boson operators was given by Schwinger [17]. However, while the classical reduced rotational system is equivalent to the reduced 1:1 resonant oscillator system, the quantization rule for the integral $j$ of each system is different. In the latter system, $n=0,1,2, \ldots$ and $j=\frac{1}{2} n$.

\section{A.5. Relative equilibria}

In this work, we work with an integrable approximation to a Hamiltonian system with three degrees of freedom and phase space $P=\mathbb{R}_{(q, p)}^{6}$. To build this approximation, we assume that the original system has an approximate dynamical Lie symmetry $G_{1: 1: 1}=\mathrm{SO}(2)$, whose action on $P$ is given by the flow $\varphi_{n}$ of the system with Hamiltonian $n$ in (A.1). The $G_{1: 1: 1}$ action on $P$ is free, and after its reduction, we obtain the first reduced system with Hamiltonian $\mathcal{H}_{n}$ on the phase space $P_{n}=\mathbb{C} P_{n}^{2}$. Each point of $P_{n}$ with $n>0$ represents (lifts to) a circular orbit $\mathbb{S}_{n}^{1}$ of $G_{1: 1: 1}$, i.e., of $\varphi_{n}$. Relative equilibria (RE) of our system are special periodic orbits in $P$, which coincide with the circular orbits $\mathbb{S}_{n}^{1}$, and which map to stationary points of $\mathcal{H}_{n}$ on $P_{n}$.

We then consider yet another dynamical symmetry $G_{1: 1}=$ $\mathrm{SO}(2)$ associated with the flow $\varphi_{n_{s}}$ of the model 1:1 resonant purely stretching Hamiltonian

$n_{s}=\frac{1}{2}\left(q_{1}^{2}+p_{1}^{2}+q_{3}^{2}+p_{3}^{2}\right)$.

Reducing second time, we arrive at the second reduced system with phase space $P_{n, n_{s}}=\mathbb{S}_{n, n_{s}}^{2}$, where $0 \leq n_{s} \leq n$, and Hamiltonian $\mathcal{H}_{n, n_{s}}$. Now, for $0<n_{s}<n$, we have RE, which lift to special dynamically invariant 2 -tori in $P$. These tori are group orbits of the total approximate dynamical symmetry $G=\mathrm{SO}(2) \times \mathrm{SO}(2)=\mathbb{S}^{2}$ induced by the combined flow $\varphi_{n} \circ \varphi_{n_{s}}$. For $n_{s}=n$ and $n_{s}=0$, we have $\mathbb{S}^{1}$ (= periodic orbit) RE. They correspond to the ones already found during the first reduction.

Alternatively, the first reduced system can have no continuous symmetries and cannot be reduced further. Periodic orbits of such system on the phase space $P_{n}$ are defined by its dynamics (see Appendix A.6), i.e., they are not group orbits. These periodic orbits lift to 2 -tori in $P$, which should be called relative periodic orbits.

\section{A.6. Dynamics of the reduced system}

Equations of motion for the reduced system are written using the Poisson algebra $\mathcal{P}$ formed by the eight invariant polynomials

$\left\{x_{2}, s_{1}, s_{2}, s_{3}, n_{3}, t_{1}, t_{2}, t_{3}\right\}$

This algebra is isomorphic to su(3). To find its structure constants we use definitions in Table A.1, compute the Poisson brackets of the above generators in the initial variables $(z, \bar{z})$ and re-express the results in terms of the integrity basis (A.2). The integral of motion $n$ is, of course, the Casimir of $\mathcal{P}$. Once the structure constants are known, the eight equations of motion are computed as brackets of the reduced Hamiltonian $H_{n}$ (an element in the enveloping algebra) and the elements of $\mathcal{P}$. An analysis of these equations is beyond the scope of our paper which concentrates solely on relative equilibria. However, in Section 4.2 we consider the dynamics restricted to the $C_{2}$-invariant subspace $\mathbb{S}^{2} \subset \mathbb{C} P^{2}$ and in Section 4.3 we study the dynamics of the second reduced system. In both cases we use an appropriate $\operatorname{so}(3)$ subalgebra of $\mathcal{P}$.

\section{Appendix B. Finite symmetries}

Since the three-atom permutations are prohibitive at the energies we consider, the total symmetry group of the vibrational Hamiltonian (2) is the $Z_{2} \times Z_{2}$ group of order four which, is generated by the bond permutation

$C_{2}:\left(\xi_{1}, \xi_{2}, \alpha, \eta_{1}, \eta_{2}, p_{\alpha}\right) \rightarrow\left(\xi_{2}, \xi_{1}, \alpha, \eta_{2}, \eta_{1}, p_{\alpha}\right)$,

and the momentum reversal

$\mathcal{T}:\left(\xi_{1}, \xi_{2}, \alpha, \eta_{1}, \eta_{2}, p_{\alpha}\right) \rightarrow\left(\xi_{1}, \xi_{2}, \alpha,-\eta_{1},-\eta_{2},-p_{\alpha}\right)$,

also known as time reversal. We describe below the consequences of the presence of this symmetry group.

The generators of the group $Z_{2} \times Z_{2}$ act on the normal mode variables as follows

$C_{2}:\left(q_{1}, q_{2}, q_{3}, p_{1}, p_{2}, p_{3}\right) \rightarrow\left(q_{1}, q_{2},-q_{3}, p_{1}, p_{2},-p_{3}\right)$,

$\mathcal{T}:\left(q_{1}, q_{2}, q_{3}, p_{1}, p_{2}, p_{3}\right) \rightarrow\left(q_{1}, q_{2}, q_{3},-p_{1},-p_{2},-p_{3}\right)$

Rewritten in terms of $(z, \bar{z})$, this action becomes

$\mathcal{T}:\left(z_{1}, z_{2}, z_{3}\right) \rightarrow\left(\bar{z}_{1}, \bar{z}_{2}, \bar{z}_{3}\right)$,

$C_{2}:\left(z_{1}, z_{2}, z_{3}\right) \rightarrow\left(z_{1}, z_{2},-z_{3}\right)$. 
Table B.1

Invariant subspaces of the action of $Z_{2} \times Z_{2}$ and its subgroups on the phase space $\mathbb{C} P^{2}$

\begin{tabular}{lll}
\hline Stabilizer & Coordinate restrictions & Topology \\
\hline$C_{2} \times \mathcal{T}$ & $\sqrt{2 n}(0,0,1)$ & Point \\
$C_{2} \times \mathcal{T}$ & $\sqrt{2 n}(\sin \theta, \cos \theta, 0), \theta=0, \ldots, 2 \pi$ & Circle $\mathbb{S}^{1}$ \\
$\mathcal{T}$ & $\left(z_{1}, z_{2}, z_{3}\right), \operatorname{Im} \mathrm{z}_{1}=\operatorname{Im} \mathrm{z}_{2}=\operatorname{Im} \mathrm{z}_{3}=0$ & $\mathbb{R} P^{2}$ \\
$C_{2}$ & $\left(z_{1}, z_{2}, 0\right)$ & $\mathbb{C} P^{1} \sim \mathbb{S}^{2}$
\end{tabular}

The $\mathbb{C} P^{2}$ restrictions on $z$ are implied.

The reduced system inherits the above finite symmetries. Taking them into account facilitates finding and analyzing relative equilibria.

\section{B.1. Action on the reduced phase space $\mathbb{C} P^{2}$}

Actions of different symmetry groups on $\mathbb{C} P^{2}$ were considered in [31] and later in [32]. Due to the presence of the $Z_{2} \times Z_{2}$ action, the phase space $\mathbb{C} P^{2}$ is not homogeneous and has several invariant subspaces which are characterized in Table B.1 and Fig. B.1. In particular, we find one fixed point with coordinates $z=\left(0,0, z_{3}\right)$. Since the phase of $z_{3}$ is irrelevant for the characterization of the point $\left(0,0, z_{3}\right)$ on $\mathbb{C} P^{2}$, we use $(0,0, \sqrt{2 n})$. We also note a $C_{2}$ invariant 2 -sphere (or $\left.\mathbb{C} P^{1}\right)$. This subspace is dynamically invariant because the spatial symmetry $C_{2}$ is symplectic. It receives our special attention in Section 4.2. We further remark that the $\mathcal{T}$-invariant real projective space $\mathbb{R} P^{2}$ and the $C_{2}$-invariant sphere $\mathbb{S}^{2}$ intersect on a circle $\mathbb{S}^{1}$, and that the fixed point $\left(0,0, z_{3}\right)$ lies on $\mathbb{R} P^{2}$.

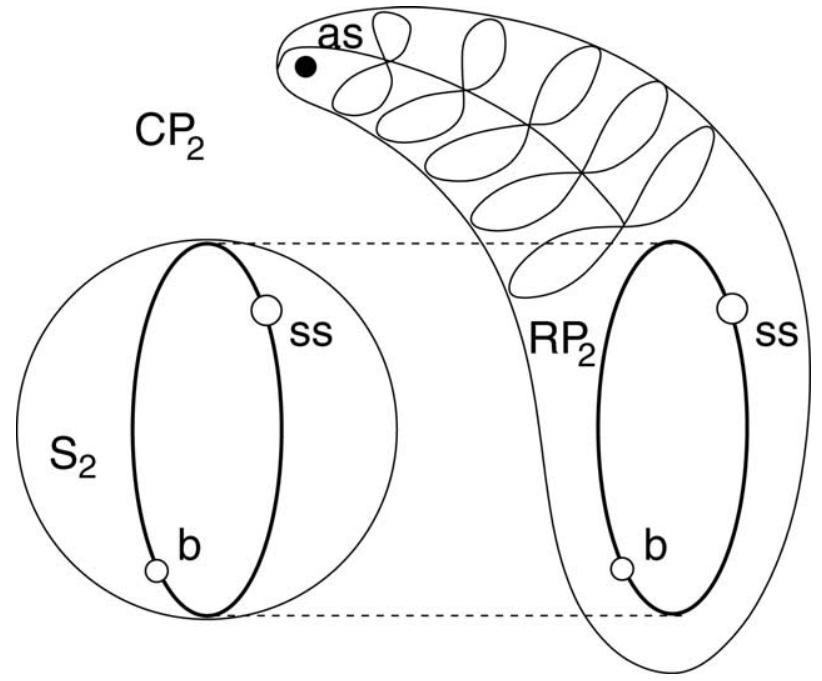

Fig. B.1. Stratification of the reduced phase space $\mathbb{C} P^{2}$ due to the action of $Z_{2} \times Z_{2}$, see Table B.1. The 2-sphere and the $\mathbb{R} P^{2}$ space are glued along the circle. Small circle dots show relative equilibria, symmetric stretch (ss), bending (b), and asymmetric stretch (as); the fixed point $(0,0,1)$ is marked black.

\section{B.2. Action on dynamical invariants}

Action of $Z_{2} \times Z_{2}$ on $\mathbb{C} P^{2}$ and resulting invariant subspaces of $\mathbb{C} P^{2}$ can be most conveniently studied using the $(z, \bar{z})$ definitions of the invariants in Table A.1. We find that

$\mathcal{T}:(n, x, s, t) \rightarrow(n, s, x,-t)$,

$C_{2}:\left(n, x, s_{3}, t_{3}\right) \rightarrow\left(n, x, s_{3}, t_{3}\right)$,

$C_{2}:\left(s_{1}, s_{2}, t_{1}, t_{2}\right) \rightarrow\left(-s_{1},-s_{2},-t_{1},-t_{2}\right)$.

In particular it follows that $\left(s_{1}, s_{2}, t_{1}, t_{2}\right)$ vanish on the $C_{2}$ invariant sphere $\mathbb{S}^{2} \in \mathbb{C} P^{2}$. Moreover, the variable $z_{3}$ vanishes on this sphere (see Table B.1) and hence

$n_{3}=s_{1}=s_{2}=t_{1}=t_{2}=0$.

Remaining invariants $\left(x_{3}, s_{3}, t_{3}\right)$ are bound by the relation

$x_{3}^{2}+s_{3}^{2}+t_{3}^{2}=n^{2}$.

We can, therefore, represent the $C_{2}$-invariant sphere embedded in an ambient space $\mathbb{R}^{3}$ with coordinates $\left(x_{3}, s_{3}, t_{3}\right)$.

We can also account for above finite symmetry properties of the dynamical invariants $(n, x, s, t)$ in order to improve the integrity basis introduced in Appendix A.3 and thus make expressing the normal form $\mathcal{H}$ even more efficient. First, since our system is invariant with respect to the time reversal operation $\mathcal{T}$ which changes sign of auxiliary invariants $\left(t_{1}, t_{2}, t_{3}\right)$, we can see immediately that $\mathcal{H}$ belongs to a smaller ring with the structure

$\mathcal{R}\left(n ; x_{3}, s_{1}, s_{2}, s_{3}\right) \times\left\{1, n_{3}, n_{3}^{2}\right\}$.

Furthermore, we can also account for the $C_{2}$ symmetry (see Appendix B) but at the cost of having a more sophisticated integrity basis

$\mathcal{R}\left(n ; x_{3}, s_{3}, s_{1}^{2}, s_{2}^{2}\right) \times\left\{1, n_{3}, n_{3}^{2}, s_{1} s_{2}, n_{3} s_{1} s_{2}, n_{3}^{2} s_{1} s_{2}\right\}$.

\section{Appendix C. Stationary points of the first normal form $\mathcal{H}_{n}$}

We use the information on symmetry and topology of the first reduced system in order to predict relative equilibria (RE) as stationary points of the first normal form $\mathcal{H}_{n}$. We then show how to find their exact positions on $\mathbb{C} P_{n}^{2}$ for a concrete $\mathcal{H}_{n}$.

\section{C.1. Prediction based on symmetry and topology}

We assume that $\mathcal{H}_{n}$ is a $Z_{2} \times Z_{2}$ invariant Morse function on $\mathbb{C} P_{n}^{2}$ whose number and type of stationary points is allowed by the topology of $\mathbb{C} P^{2}$ and the symmetry $Z_{2} \times Z_{2}$. The action of $Z_{2} \times Z_{2}$ on $\mathbb{C} P^{2}$ is detailed in Appendix B. This action has the isolated fixed point $(0,0, \sqrt{2 n})$ (critical orbit) which must be the stationary point of $\mathcal{H}_{n}$. Furthermore, $\mathcal{H}_{n}$ should obey Morse requirements for $\mathbb{C} P^{2}$ and for each 
invariant subspace in Table B.1. In particular recall that the numbers $c_{k}$ of stationary points of Morse index $k$ of a Morse function $\mathcal{H}_{n}$ on $P$ obey the Euler equality

$\sum(-1)^{k} c_{k}=\sum(-1)^{k} b_{k}$

where $b_{k}$ are Betti numbers for $P$. Consulting the Betti numbers $b_{k}$ and the Euler characteristics $\sigma=\sum(-1)^{k} b_{k}$ of the subspaces listed below,

\begin{tabular}{lllllll}
\hline Space & $b_{0}$ & $b_{1}$ & $b_{2}$ & $b_{3}$ & $b_{4}$ & $\sigma$ \\
\hline $\mathbb{C} P^{2}$ & 1 & 0 & 1 & 0 & 1 & 3 \\
$\mathbb{R} P^{2}$ & 1 & $0_{2}$ & 0 & & & 1 \\
$\mathbb{S}^{2}$ & 1 & 0 & 1 & & & 2 \\
$\mathbb{S}^{1}$ & 1 & 1 & & & & 0 \\
\hline
\end{tabular}

we conclude that $\mathcal{H}_{n}$ should have at least three stationary points on $\mathbb{C} P^{2}$, two on $\mathbb{S}^{2}$, and two on $\mathbb{S}^{1}$.

Note that, the $\mathbb{R} P^{2}$ space has Euler characteristics 1. [This space is non-orientable, its first homology group $H_{1}$ is $Z_{2}$ and $H_{2}$ is trivial, so that ranks $b_{1}=b_{2}=0$.]. A Morse function on $\mathbb{R} P^{2}$ should have at least three stationary points.

All these requirements can be satisfied by the three points representing symmetric stretch (ss), asymmetric stretch (as), and bending (b) relative equilibria. The as RE is a fixed point, while ss and $\mathrm{b}$ lie on the circle $\mathbb{S}^{1}=\mathbb{S}^{2} \cap \mathbb{R} P^{2}$ as shown in Fig. B.1. All RE have time reversal symmetry; ss and $b$ are also $C_{2}$-invariant. This minimal $\mathrm{RE}$ set is further confirmed by the stability analysis.

\section{C.2. Position of stationary points}

When energies are close to 0 (near the equilibrium $z=0$ ) and $n$ is small the ss and $\mathrm{b}$ points correspond to the symmetric stretch and bending normal modes with coordinates $(\sqrt{2 n}, 0,0)$ and $(0, \sqrt{2 n}, 0)$, respectively. To find the position of ss and $\mathrm{b}$ we write equations of motion on $\mathbb{C} P^{2}$ and restrict them to the $C_{2}$-invariant sphere $\mathbb{S}^{2}$ (see Section A.4). The three equations remaining after such restriction are written in terms of the so(3) algebra generated by $\left(x_{3}, s_{3}, t_{3}\right)$. They resemble Euler's equations. We further restrict these equations to the $\mathcal{T}$-invariant circle $\mathbb{S}^{1}$ by setting $t_{3}=0$. The Hamiltonian $\mathcal{H}_{n}$ is $\mathcal{T}$-invariant and does not depend on $t_{3}$. As a result, $\dot{x}_{3}$ and $\dot{s}_{3}$ are multiples of $t_{3}$ and vanish. To solve the last equation

$$
\frac{\mathrm{d} t_{3}}{\mathrm{~d} t}=\left\{t_{3}, H_{n}\right\}=417.7450 s_{3}+f_{n}\left(x_{3}, s_{3}\right) \epsilon^{2}+\cdots=0,
$$

where

$$
\begin{aligned}
f_{n}= & 2.0902\left(x_{3}^{2}-s_{3}^{2}\right)-3.7947 n s_{3}+2.6046 x_{3} s_{3} \\
& +4.6048 x_{3} n,
\end{aligned}
$$

we use the 2-sphere restriction (B.1) with $t_{3}=0$, and recall that at small $n$, the value of $x_{3}$ equals approximately $n$ for the ss point and $-n$ for the $\mathrm{b}$ point while $s_{3}$ is close to 0 . Then

$x_{3}= \pm n\left(1-\frac{1}{2} \frac{s_{3}^{2}}{n^{2}}-\frac{1}{8} \frac{s_{3}^{4}}{n^{4}}-\cdots\right)$.

The formal series solution for $s_{3}$ and $x_{3}$ is obtained by Newton's iteration and is shown in Table 4.

\section{C.3. Stability of $R E$}

Given the normal mode frequencies $\omega_{1}>\omega_{3}>\omega_{2}$ of ozone and the Morse requirements for $\mathbb{C} P^{2}$ and the subspaces involved, we can further infer that in the absence of any other stationary points the signatures should be $(----)$ (Morse index 4), $(++--)$ (index 2), and $(++++)$ (index 0$)$, respectively. The ss and $\mathrm{b}$ points remain stable when restricted on $\mathbb{S}^{2}$, where their signatures are (- - ) (maximum, index 2$)$ $(++)$ (minimum, index 0), respectively. The Morse index of ss and $\mathrm{b}$ on $\mathbb{R} P^{2}$ is also 0 and 2 , while the as point on $\mathbb{R} P^{2}$ is unstable with signature $(-+)$ and index 1 . In general, Hamiltonian stability of RE cannot be found from Morse indexes. It is clear, however, that ss and $b$ remain stable (elliptic) as long as they correspond to the global maximum and minimum of energy at given $n$.

To check the stability of the as RE in the limit $n \rightarrow 0$, we rewrite the lowest order of the first normal form (cf. Table A.1)

$$
\begin{aligned}
H_{n}^{(0)} & =923.488 n+208.872 x_{3}+163.455 n_{3} \\
& \approx 1087 n+45 n_{1}-372 n_{2},
\end{aligned}
$$

as a local linearized Hamiltonian $\omega_{1}^{\prime} n_{1}+\omega_{2}^{\prime} n_{2}$ near the fixed point (as) with $z=(0,0, \sqrt{2 n})$. We conclude that at very low $n$ the (as) RE is stable (elliptic) with one small positive frequency $\omega_{1}^{\prime}$ and one large negative frequency $\omega_{2}^{\prime}$. To find how $\omega_{1}^{\prime}$ and $\omega_{2}^{\prime}$ change with $n$, we can use $\left(z_{1}, z_{2}\right)$ as local coordinates near $z=(0,0, \sqrt{2 n})$, express

$z_{3}=\bar{z}_{3}=\sqrt{2 n-z_{1} \bar{z}_{1}-z_{2} \bar{z}_{2}}$,

and linearize higher order terms $\mathcal{H}_{n}\left(z_{1}, z_{2}, \bar{z}_{1}, \bar{z}_{2}\right)$ from Table 3. The eigenvalues of the Hamiltonian matrix of this linearization are

$\lambda_{1}^{2}=-45^{2}+668.97 n+475.81 n^{2}-14.517 n^{3}+\cdots$,

$\lambda_{2}^{2}=-372^{2}+11465.5 n-190.89 n^{2}+29.756 n^{3}+\cdots$.

It can be seen that $\lambda_{2}$ remains imaginary within the interval $n=0, \ldots, 10$ of the validity of our normal form, while $\lambda_{1}$ becomes quickly real at $n \approx 1.50$. This bifurcation corresponds to the normal-to-local mode transition described as point A in Section 3.2 (see Fig. 1). It is further analyzed using the second normal form in Section 4.3 (see Fig. 4) and 
Appendix D.3. The value of 1.50 agrees well with 1.24 obtained in Section 3.2.

Detailed analysis of the equations of motion on $\mathbb{C} P_{n}^{2}$ can also provide Hamiltonian stability of the ss and $b$ RE, as well as of the local mode RE. This, however, requires extensive calculations because their coordinates on $\mathbb{C} P^{2}$ are not fixed. Examples of such analysis can be found in [33d,c,].

\section{Appendix D. Second normal form $\mathcal{H}_{n, n_{s}}$}

The first reduced system has two degrees of freedom. We can further normalize this system, if a second approximate integral of motion $n_{s}=n_{1}+n_{3}$ in (A.3) is introduced. This new approximation reflects the fact that the ratio of frequencies of the two stretching modes of ozone $\omega_{1}$ and $\omega_{3}$ is very close to 1:1 (see Section 4.3).

\section{D.1. Invariants and integrity basis}

Like in the case of the hyperpolyad integral $n$, we consider the new dynamical symmetry induced by the flow

$\varphi_{n_{s}}:\left(z_{1}, z_{2}, z_{3}, t\right) \rightarrow\left(\mathrm{e}^{\mathrm{i} t} z_{1}, z_{2}, \mathrm{e}^{\mathrm{i} t} z_{3}\right)$

of the system with Hamiltonian $n_{s}$. This flow is a rotation in $\mathbb{C}^{3}$. We normalize the first normal form $\mathcal{H}_{n}$ with regard to this symmetry and obtain the second normal form $\mathcal{H}_{n, n_{s}}$ whose terms Poisson commute with both $n$ and $n_{s}$. This further restricts the set of generators in Table A. 1 to those, whose monomials have the same degree in $\left(z_{1}, z_{3}\right)$ and $\left(\bar{z}_{1}, \bar{z}_{3}\right)$. When selecting invariants, we should, of course, pick $n$ and $n_{s}$. Then we are left with three linearly independent invariants $x_{2}, s_{2}$, and $t_{2}$, which are bound by the algebraic relation

$x_{2}^{2}+s_{2}^{2}+t_{2}^{2}=n_{s}^{2}$.

It can be shown that these invariants generate the ring of all $\varphi_{n}$ and $\varphi_{n_{s}}$ invariant polynomials with the structure

$\mathcal{R}\left(n, n_{s} ; x_{2}, s_{2}\right) \times\left\{1, t_{2}\right\}$.

Furthermore, since the second reduced Hamiltonian $\mathcal{H}_{n, n_{s}}$ is $Z_{2} \times Z_{2}$-invariant, it belongs to a smaller ring

$\mathcal{R}\left(n, n_{s} ; x_{2}, s_{2}^{2}\right)$,

cf. Appendix B.2. This ring is freely generated by $x_{2}$ and $s_{2}^{2}$. In other words, $\mathcal{H}_{n, n_{s}}$ can be considered as a function of two variables $\left(x_{2}, s_{2}^{2}\right)$ and, of course, parameters $\left(n, n_{s}\right)$.

\section{D.2. Reduced phase space and its stratification}

Relation (D.1) proves that (for any $n_{s}>0$ ) the reduced phase space of the second reduced system is yet another 2sphere $\mathbb{S}_{n_{s}}^{2}$ (often called polyad sphere [12]), which can be defined by (D.1) in the ambient space $\mathbb{R}^{3}$ with coordinates $\left(x_{2}, s_{2}, t_{2}\right)$. The action of the finite symmetry group $Z_{2} \times Z_{2}$ on this $\mathbb{R}^{3}$ space

$C_{2}:\left(x_{2}, s_{2}, t_{2}\right) \rightarrow\left(x_{2},-s_{2},-t_{2}\right)$,

$\mathcal{T}:\left(x_{2}, s_{2}, t_{2}\right) \rightarrow\left(x_{2}, s_{2},-t_{2}\right)$,

can be easily found using Appendix B.2. It can be seen that $Z_{2} \times Z_{2}$ acts on $\mathbb{R}^{3}$ and on the sphere $\mathbb{S}_{n_{s}}^{2} \subset \mathbb{R}^{3}$ as a point group of transformations $C_{2 v}$ with its axis $C_{2}$ oriented as axis $x_{2}$. The action of this group on $\mathbb{S}_{n_{\mathrm{s}}}^{2}$ has two fixed points at the north and south poles, see Fig. 4, left, which form two one-point critical orbits. The reflection planes $\mathcal{T}$ and $C_{2} \circ \mathcal{T}$ intersect $\mathbb{S}_{n_{s}}^{2}$ on two circles, each circle minus two critical orbits forms a one-dimensional stratum.

\section{D.3. Dynamics and stationary points}

A Morse function $\mathcal{H}_{n, n_{s}}$ on $\mathbb{S}^{2}$ should have at least two stationary points, a maximum and a minimum. In our case, the two fixed points of the $Z_{2} \times Z_{2}$ symmetry group action are necessarily the stationary points of $\mathcal{H}_{n, n_{s}}$. Their coordinates are

$x_{2}= \pm n_{s}, \quad s_{2}=t_{2}=0$.

When $n_{s}=n$ (i.e., $\delta=0$ ), these points correspond to the symmetric ss $\left(x_{2}=-n_{s}\right)$ and asymmetric as $\left(x_{2}=n_{s}\right)$ stretch RE. This can be verified directly using the expression for $\mathcal{H}_{n, n_{s}}$ at these points given in Table 6. In the limit $n_{s}=0$ (i.e., $\delta=n$ ) both points correspond to the bending RE b.

The concrete function $H_{n, n_{s}}$ may, of course, have more stationary points. The latter can lie either on one-dimensional strata or on the generic stratum. If such points bifurcate from ss or b, they, typically, should depart on a one-dimensional stratum (circles in Fig. 4, left). To find new stationary points, we should consider equations of motion for $\left(x_{2}, s_{2}, t_{2}\right)$. The Poisson algebra generated by invariants $\left(\frac{1}{2} x_{2}, \frac{1}{2} s_{2}, \frac{1}{2} t_{2}\right)$ is a standard so(3) algebra with Casimir $n_{s}$. Since $\mathcal{H}_{n, n_{s}}$ is $\mathcal{T}$ invariant, it does not depend on $t_{2}$, so that $\dot{x}_{2}$ and $\dot{s}_{2}$ vanish when $t_{2}=0$. Therefore, all time reversal invariant stationary solutions (with $t_{2}=0$, i.e., on the $\mathcal{T}$ stratum) other than those at the fixed points (with $s_{2}=0$ ) satisfy the equation

$\left\{t_{2}, \mathcal{H}_{n, n_{s}}\right\} s_{2}^{-1}=a x_{2}^{2}+b x_{2}+c=0$,

and, of course,

$x_{2}^{2}+s_{2}^{2}=n_{s}^{2} \leq n^{2}$.

From Table 5 we obtain

$a=-0.41839 \epsilon^{4}$,

$b=40.775 \epsilon^{2}+\left(2.37859 n-1.02332 n_{s}\right) \epsilon^{4}$, 


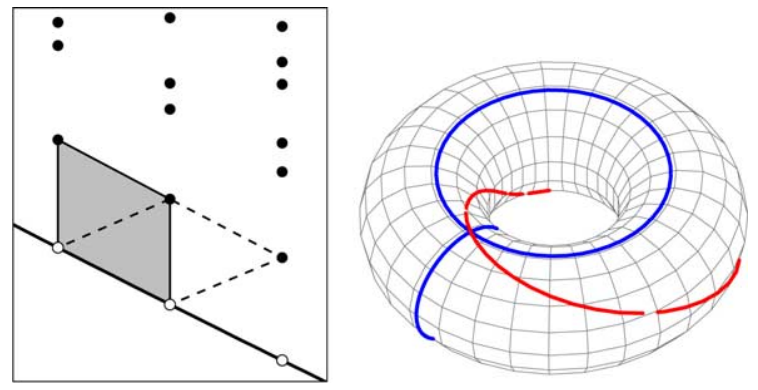

Fig. D.1. Left: fragment of the diagram in Fig. 2 with the elementary cell of the local quantization in Section 3.3 (dashed line) and the polyad quantization in Section 3.4 (solid line and shaded area). Filled circles show quantum states with $N=3,4$, and 5; opaque circles mark classical RE limits; thick solid line at the minimum energy corresponds to the bending RE. Right: corresponding cycle bases on the EBK torus.

$$
\begin{aligned}
c & =-45.417+\left(2.1308 n_{s}-8.0594 n\right) \epsilon^{2} \\
& +\left(0.39487 n n_{s}-0.20549 n^{2}+0.20054 n_{s}^{2}\right) \epsilon^{4} .
\end{aligned}
$$

(Note that $\epsilon$ is a placeholder for the formal smallness parameter and should be set to 1.) Solving these equations we confirm that at very low values of $n$ and $n_{s}$ the second normal form $H_{n, n_{s}}$ is the simplest Morse function on $\mathbb{S}^{2}$ with only two stationary points, a maximum at $x_{2}=-n_{s}$ and a minimum at $x_{2}=n_{s}$. Additional pair of equivalent solutions is characterized in Table 7. This pair appears when $n$ becomes larger than

$n_{\text {crit }}=1.24438+1.13419 \delta-0.35633 \times 10^{-2} \delta^{2}+\cdots$,

where $n \geq \delta=n-n_{s} \geq 0$. (Notice the excellent agreement with the value of $n_{\text {crit }}$ for the $A$ point in Section 3.2.) The two new RE are, of course, the local modes. The normal-tolocal mode bifurcation at $n=n_{\text {crit }}$ is a textbook example of a bifurcation with broken symmetry $Z_{2}$ (which in our case is realized as $C_{2} \circ \mathcal{T}$ or $C_{2}$ ); it is also often called a "pitchfork" bifurcation. As $n>n_{\text {crit }}$ increases the two points move along the $\mathcal{T}$-invariant circle where $t_{2}=0$. The $C_{2}$ symmetry makes these points equivalent; they are absolute minima of $\mathcal{H}_{n, n_{s}}$ (see Fig. 4, right).

\section{Appendix E. On the choice of local action-angle coordinates in different EBK quantization schemes}

In Sections 3.3, 3.4, 4.4, and 4.5, we used different Einstein-Brillouin-Keller (EBK) quantization schemes in order to describe all or part of the quantum states of our system. It is instructive to understand the difference of these schemes. Recall that EBK quantization uses local action-angle coordinates, which are defined by the choice of the local cycle basis on the EBK tori. In Section 3.3, one of the cycles $\gamma_{0}$ follows the periodic orbit (PO), and two others $\left(\gamma^{\prime}, \gamma^{\prime \prime}\right)$ correspond to small oscillations about the PO. Recall that this PO is a relative equilibrium and is the orbit of the flow $\varphi_{n}$. Conse- quently, exciting transversal modes with quantum numbers $\left(n^{\prime}, n^{\prime \prime}\right)$ changes the hyperpolyad number $n$. The cycle basis for the same torus in the polyad scheme of Section 3.4 is $\left(\gamma_{0}, \gamma^{\prime}-\gamma_{0}, \gamma^{\prime \prime}-\gamma_{0}\right)$. As a consequence, local quantum numbers in the two schemes differ as illustrated in Fig. D.1.

Quantizing the stretching polyad action $n_{s}$ in Section 4.4 has some similarity with that for the bending RE in Section 3.3, cf. Figs. D.1 and 5. The difference now is that $\omega^{\prime}$ and $\omega^{\prime \prime}$ are assumed to be in 1:1 resonance and therefore, $n^{\prime}$ and $n^{\prime \prime}$ cannot be used as independent conserved quantities. We use the "small polyad" number $n_{s}=n^{\prime}+n^{\prime \prime}$ instead.

\section{References}

[1] (a) Vl.I. Arnol'd, Matematicheskie Metody Klassicheskoí Mekhaniki, Nauka, Moscow, 1974 (in Russian); Mathematical Methods of Classical Mechanics, translated by K. Vogtmann and A. Weinstein, 2nd ed., Ser. Graduated Texts in Mathematics 60, Springer-Verlag, New York, 1989 ;

(b) Vl.I. Arnol'd, V.V. Kozlov, A.I. Neíshtadt, Mathematical Aspects of Classical and Celestial Mechanics, Dynamical Systems. III Encyclopedia of Mathematical Sciences, vol. 3, Springer-Verlag, Berlin, 1988.

[2] J. Montaldi, R.M. Roberts, J. Nonlin. Sci. 9 (1999) 53-88.

[3] A. Dorney, J.K.G. Watson, J. Mol. Spectrosc. 42 (1972) 135-148.

[4] W.G. Harter, Molecular symmetry and dynamics, in: G.W.F. Drake (Ed.), Atomic, Molecular, and Optical Physics Handbook, AIP Press, New York, 1996, pp. 378-393 and references therein.

[5] M.S. Child, in: P. Jensen, P.R. Bunker (Eds.), Computational Molecular Spectroscopy, Wiley, Chichester, 2000.

[6] W.G. Harter, C.W. Patterson, J. Chem. Phys. 66 (1977) 4872-4885; W.G. Harter, C.W. Patterson, J. Chem. Phys. 66 (1977) 4886-4892; W.G. Harter, C.W. Patterson, J. Math. Phys. 20 (7) (1979) 1453-1459; W.G. Harter, C.W. Patterson, J. Chem. Phys. 80 (1984) 4241-4261.

[7] Vl.I. Arnol'd, Geometrical Methods in the Theory of Ordinary Differential Equations, translated by J. Szücs, English translation edited by M. Levi, Comprehensive Studies in Mathematics 250, Springer-Verlag, New York, 1988.

[8] (a) W. Gröbner, Die Lie-Reihen und ihre Anwendungen, Ser. Mathematische Monographien, vol. 3, Deutscher Verlag der Wissenschaften, Berlin, 1960;

(b) W. Gröbner, Contributions to the method of Lie series, in: W Gröbner, H. Knapp (Eds.), Ser. B. I. Hochschuleskripten, vol. 802/802a, Bibliographisches Institute, Mannheim, 1967;

(c) A. Deprit, Celest. Mech. 1 (1969) 12-30

(d) J. Henrard, Celest. Mech. 3 (1970) 107-120.

[9] R.H. Cushman, D. Rod, Physica D 6 (1982) 105-112.

[10] R.H. Cushman, Proc. R. Soc. London, Ser. A 382 (1982) 361-371; R.C. Churchill, M. Kummer, D.L. Rod, J. Diff. Eqns. 71 (1983) 359_ 414.

[11] N. Fulton, J. Tennyson, D.A. Sadovskií, B.I. Zhilinskií, J. Chem. Phys. 99 (1993) 906-918.

[12] L. Xiao, M.E. Kellman, J. Chem. Phys. 90 (1989) 6086-6098.

[13] D.A. Sadovskií, B.I. Zhilinskií, J. Chem. Phys. 103 (1995) 10520 10536.

[14] Z.-M. Lu, M.E. Kellman, J. Chem. Phys. 107 (1997) 1-15.

[15] (a) V1.G. Tyuterev, S. Tashkun, P. Jensen, A. Barbe, T. Cours, J. Mol Spectrosc. 198 (1) (1999) 57-76;

(b) Vl.G. Tyuterev, S.A. Tashkun, D.W. Schwenke, P. Jensen, T. Cours, A. Barbe, M. Jacon, Chem. Phys. Lett. 316 (3/4) (2000) 271-279; (c) A. Barbe, A. Chichery, T. Cours, V1.G. Tyuterev, J.J. Plateaux, J. Mol. Struct. 616 (1-3) (2002) 55-65. 
[16] G. Herzberg, Molecular Spectra and Molecular Structure. I. Spectra and Structure of Polyatomic Molecules, R.E. Krieger, Florida, 1989.

[17] J. Schwinger, On angular momentum, in: L.C. Biedenharn, H. Van Dam (Eds.), Quantum Theory of Angular Momentum, Academic Press, New York, 1975, pp. 229-279.

[18] E.B. Wilson Jr., J.C. Decius, P.C. Cross, Molecular Vibrations, McGraw-Hill, New York, 1955.

[19] A. Weinstein, Invent. Math. 20 (1973) 47-57.

[20] (a) J. Montaldi, R.M. Roberts, I. Stewart, Phil. Trans. R. Soc. London Ser. A 325 (1988) 237-293;

(b) J. Montaldi, R.M. Roberts, I. Stewart, Nonlinearity 3 (1990) 695730 ;

J. Montaldi, R.M. Roberts, I. Stewart, Nonlinearity 3 (1990) 730-772; (c) J. Montaldi, Nonlinearity 10 (1997) 449-466.

[21] R.T. Lawton, M.S. Child, Mol. Phys. 37 (1979) 1799; Ch. Jaffé, P. Brumer, J. Chem. Phys. 73 (1980) 5646; M.S. Child, L. Halonen, Adv. Chem. Phys. 57 (1984) 1; I.M. Mills, A.G. Robiette, Mol. Phys. 56 (1985) 743.

[22] V1.G. Tyuterev, Private communication, February 2000.

[23] L.E. Fried, G.S. Ezra, J. Chem. Phys. 86 (1987) 6270-6282; Ch. Jaffé, J. Chem. Phys. 89 (1988) 3395-3396; M.E. Kellman, J. Chem. Phys. 93 (1990) 6630-6635; M.E. Kellman, G. Chen, J. Chem. Phys. 95 (1991) 8671-8672.

[24] (a) C. Jaffé, W.P. Reinhardt, J. Chem. Phys. 71 (4) (1979) 18621869 ;

C. Jaffé, W.P. Reinhardt, J. Chem. Phys. 77 (10) (1982) 5191-5203;

(b) K. Stefanski, E. Pollak, J. Chem. Phys. 87 (2) (1987) 1079-1088;

(c) A.B. McCoy, E.L. Sibert, Mol. Phys. 77 (4) (1992) 697-708;

A.B. McCoy, E.L. Sibert, Mol. Phys. 95 (5) (1991) 3476-3487;

A.B. McCoy, E.L. Sibert, Mol. Phys. 97 (5) (1992) 2938-2947;

(d) A.B. McCoy, E.L. Sibert, Mol. Phys. 105 (1996) 469-478;

(e) M. Joyeux, J. Chem. Phys. 109 (6) (1998) 2111-2122;

(f) X.-G. Wang, E.L. Sibert, J. Chem. Phys. 113 (13) (2000) 5384-5400.

[25] F. Pérez-Bernal, J.M. Arias, A. Frank, R. Lemus, R. Bijker, J. Mol. Spectrosc. 184 (1997) 1-11.

[26] R. Siebert, P. Fleurat-Lessard, R. Schinke, M. Bittererova, S.C. Farantos, J. Chem. Phys. 116 (22) (2002) 9749-9767.

[27] M. Joyeux, Private communication.
[28] R. Prosmiti, S.C. Farantos, J. Chem. Phys. 103 (9) (1995) 3299-3314.

[29] I.N. Kozin, R.M. Roberts, J. Tennyson, J. Chem. Phys. 111 (1999) $140-150$.

[30] CONTENT 1.5 by Yu.A. Kuznetsov et al., see ftp://ftp. cwi.nl/pub/CONTENT/ and http://www. math.uu.nl/people/kuznet/.

[31] B.I. Zhilinskií, Chem. Phys. 137 (1989) 1-13.

[32] D.A. Sadovskií, B.I. Zhilinskií, Phys. Rev. A 48 (1993) 1035-1044.

[33] (a) Ch. Van Hecke, D.A. Sadovskií, B.I. Zhilinskií, V. Boudon, European Phys. J. D 17 (2001) 13-35;

(b) K. Efstathiou, R.H. Cushman, D.A. Sadovskií, Proc. R. Soc. London Ser. A 459 (2040) (2003) 2997-3019;

(c) K. Efstathiou, D.A. Sadovskí, Nonlinearity 17 (2004) 415-446;

(d) K. Efstathiou, D.A. Sadovskií, B.I. Zhilinskií, SIADS 3 (2004) 261-351.

[34] R.H. Cushman, L. Bates, Global Aspects of Classical Integrable Systems, Birkhäuser, Basel, 1997.

[35] Y. Colin de Verdier, S. Vũ Ng̣̣c, Ann. Ec. Norm. Sup. 36 (2003) 155.

[36] H. Broer, I. Hoveijn, G. Lunter, G. Vegter, Bifurcations in Hamiltonian systems. Computing singularities by Gröbner bases, in: J.-M. Morel, F. Takens, B. Teissier (Eds.), Ser. Lecture Notes in Mathematics, vol. 1806, Springer-Verlag, Berlin, 2003.

[37] J.F. Svitak, V. Tyng, M.E. Kellman, J. Phys. Chem. A 106 (45) (2002) 10797-10805.

[38] I. Dolgachev, Weighted projective varieties, in: Group Actions and Vector Fields, Ser. Lecture Notes in Mathematics, vol. 966, SpringerVerlag, Berlin, 1982, pp. 34-71.

[39] S. Egilsson, On embedding the 1:1:2 resonance space in a Poisson manifold. Preprint, Department of Mathematics, University of California, Berkeley, 1995; also appeared in Electronic Res. Announc. Am. Math. Soc. 1 (1995) 48-56.

[40] (a) H. Dullin, A. Giacobbe, R.H. Cushman, Physica D 190 (2003) 1537 ;

(b) R.H. Cushman, H.R. Dullin, A. Giacobbe, D.D. Holm, M. Joyeux, P. Lynch, D.A. Sadovskií, B.I. Zhilinskií, Phys. Rev. Lett. (2004) 0243021-4;

(c) A. Giacobbe, R.H. Cushman, D.A. Sadovskií, B.I. Zhilinskií, J. Math. Phys., in press. 